DSF-93/52

MIT-CTP-2286

\title{
PROTON DECAY AND NEUTRINO MASSES IN SO(10)
}

\author{
F. Acampora*, \\ G. Amelino-Camelia ${ }^{\triangle}$, \\ F. Buccella ${ }^{\dagger *}$, \\ O. Pisanti ${ }^{\dagger}$, \\ L. Rosa ${ }^{\dagger \ddagger}$ and \\ T. Tuzi* \\ * Istituto di Fisica Teorica, Mostra d'Oltremare, 80125 Napoli, Italia. \\ $\triangle$ Center for Theoretical Physics, Laboratory of Nuclear Science and Department of \\ Physics, Massachusetts Institute of Technology, Cambridge, Massachusetts 02139, USA. \\ † Dipartimento di Scienze Fisiche, Mostra d'Oltremare Pad. 19, 80125 Napoli, Italia. \\ ‡ Istituto Nazionale di Fisica Nucleare, Mostra d'Oltremare Pad. 20, 80125 Napoli, Italia.
}

\begin{abstract}
In the last few years physicists have been looking at $S O(10)$ GUT models with renewed attention because it has been realized that the SU(5) minimal model cannot unify the strong, electromagnetic and weak interactions in a way consistent with the experimental values of $\alpha\left(M_{Z}\right), \sin ^{2} \theta_{W}\left(M_{Z}\right)$ and $\alpha_{S}\left(M_{Z}\right)$. In this paper we derive lower limits on neutrino masses, relevant for cosmology and for the solar-neutrino problem, from necessary consistency conditions on a class of $S O(10)$ models with $S U(4)_{P S} \otimes S U(2)_{L} \otimes S U(2)_{R}$ or $S U(3)_{c} \otimes S U(2)_{L} \otimes S U(2)_{R} \otimes U(1)_{B-L}$ intermediate gauge symmetry.
\end{abstract}

\section{CONTENTS}

I. Introduction

II. Symmetry Breaking for Various Higgs Potentials

A. $G^{\prime} \equiv S U(4)_{P S} \otimes S U(2)_{L} \otimes S U(2)_{R} \times D$

B. The 210 representation

1. $G^{\prime} \equiv S U(4)_{P S} \otimes S U(2)_{L} \otimes S U(2)_{R}$

2. $G^{\prime} \equiv S U(3)_{c} \otimes S U(2)_{L} \otimes S U(2)_{R} \otimes U(1)_{B-L} \times D$

3. $G^{\prime} \equiv S U(3)_{c} \otimes S U(2)_{L} \otimes S U(2)_{R} \otimes U(1)_{B-L}$

III. Renormalization-Group Equations
A. $G^{\prime} \equiv S U(4) \otimes S U(2) \otimes S U(2) \times D$
B. $G^{\prime} \equiv S U(4)_{c} \otimes S U(2)_{L} \otimes S U(2)_{R}$
C. $G^{\prime} \equiv S U(3)_{c} \otimes S U(2)_{L} \otimes S U(2)_{R} \otimes U(1)_{B-L} \times D$
D. $G^{\prime} \equiv S U(3)_{c} \otimes S U(2)_{L} \otimes S U(2)_{R} \otimes U(1)_{B-L}$
IV. Conclusions

Appendix

References 


\section{Introduction}

A $S O(10)$ (Georgi, 1975; Fritzsch and Minkowski, 1975) unified model with intermediate symmetry $S U(4)_{P S} \otimes S U(2)_{L} \otimes S U(2)_{R} \equiv G_{422}$ (Pati and Salam, 1974; Chang, Mohapatra, and Parida, 1984; Chang, Gipson, Marshak, Mohapatra, and Parida, 1985), broken at a scale $M_{R} \sim 10^{11} \mathrm{GeV}$, has been recently advocated (Mohapatra and Parida, 1993; Babu and Shafi, 1993) to give, through the see-saw mechanism (Gell-Mann, Ramond, and Slansky, 1980; Yanagida, 1979), neutrino masses of the order required to explain the solar-neutrino problem within the framework of the MSW theory, and to account for at least part of the dark matter in the universe (Schramm, 1992).

$S O(10)$ unified models have been studied since many years with the physical motivation of obtaining values for the masses of the lepto-quarks which mediate proton decay higher than the ones found within the $S U(5)$ minimal model. The $S U(5)$ predictions for these masses are too low with respect to the experimental lower limit on proton decay: $\tau_{p \rightarrow e^{+} \pi^{0}} \geq 9 \cdot 10^{32}$ years (Review of Particle Properties, 1992), which corresponds (le Yaouanc, Oliver, Pène, and Raynal, 1977) to the following lower limit on the masses (which we indicate with $M_{X}$ ) of the lepto-quarks which mediate that decay:

$$
M_{X} \geq 3.2 \cdot 10^{15} \mathrm{GeV} \text {. }
$$

Figure 1: Evolution of the three coupling constants 
Recently, the more precise determination of the gauge coupling constants at the scale $M_{Z}$ has allowed to show that, if only standard model particles contribute to the RenormalizationGroup Equations (RGE), the three running coupling constants of $G \equiv S U(3) \otimes S U(2) \otimes U(1)$ meet at three different points (Amaldi, de Boer, and Furstenau, 1991) [fig.1] and only the meeting point of $\alpha_{2}(\mu)$ and $\alpha_{S}(\mu)$ corresponds to a value of the scale $\mu$ sufficiently high to comply with the experimental lower limit on proton decay.

Indeed it has been observed (Amelino-Camelia, Buccella, and Rosa, 1990) that $S O(10)$, for which the hypercharge is the combination of two generators belonging to its Cartan,

$$
Y=T_{3 R}+\frac{B-L}{2}
$$

is very promising to modify the $\mathrm{SU}(5)$ predictions in such a way to prevent conflict with experiment. In fact, if there is an intermediate symmetry group $G^{\prime}$ containing $S U(2)_{R}$ and/or $S U(4)_{P S}$, to which $T_{3 R}$ and $B-L$ belong respectively, the non-Abelian evolution of either component of $\mathrm{Y}$ implies a higher unification point.

An analysis of the possible symmetry breaking patterns with Higgses in representations with dimension $\leq 210$ and with only an intermediate symmetry group $G^{\prime}$ between $G$ and $S O(10)$ led to four different possibilities for $G^{\prime}$ (Buccella, 1988), which are reported in table I together with the Higgs which must take the highest Vacuum Expectation Value (VEV) $\left(\sim M_{X}\right)$ in order to have spontaneous breaking of $S O(10)$ to $G^{\prime} \cdot G^{\prime}$ is then spontaneously broken to $G$ at the scale $M_{R}$ by an Higgs in the representation $126 \oplus \overline{126}$. In table I, $D$ is the left-right discrete symmetry, which interchanges $S U(2)_{L}$ and $S U(2)_{R}$, first introduced by Kuzmin and Shaposnikov (Kuzmin and Shaposhnikov, 1980).

For the models in table I, using one-loop approximation for the RGE, one obtains the following upper limit for the value of the scale of $S O(10)$-breaking (Buccella, Miele, Rosa, Santorelli, and Tuzi, 1989):

$$
M_{X} \leq M_{Z} \exp \frac{\pi}{2} \frac{\left(\sin ^{2} \theta_{W}\left(M_{Z}\right)-\frac{\alpha}{\alpha_{S}}\left(M_{Z}\right)\right)}{\alpha\left(M_{Z}\right)} .
$$

An upper limit, that is more restrictive by a factor of about $\frac{1}{3}$, is obtained using a two-loop approximation (Tuzi, 1989). 
TABLE I

\begin{tabular}{|c|c|c|}
\hline$G^{\prime}$ & Higgs direction & Representation \\
\hline$S U(4)_{P S} \otimes S U(2)_{L} \otimes S U(2)_{R} \times D$ & $\omega_{L}=\frac{2\left(\omega_{11}+\ldots+\omega_{66}\right)-3\left(\omega_{77}+\ldots \omega_{00}\right)}{\sqrt{60}}$ & 54 \\
\hline$S U(4)_{P S} \otimes S U(2)_{L} \otimes S U(2)_{R}$ & $\Phi_{T}=\Phi_{7890}$ & 210 \\
\hline$S U(3)_{c} \otimes S U(2)_{L} \otimes S U(2)_{R} \otimes U(1)_{B-L} \times D$ & $\Phi_{L}=\frac{\Phi_{1234}+\Phi_{1256}+\Phi_{3456}}{\sqrt{3}}$ & 210 \\
\hline$S U(3)_{c} \otimes S U(2)_{L} \otimes S U(2)_{R} \otimes U(1)_{B-L}$ & $\Phi(\theta)=\cos \theta \Phi_{L}+\sin \theta \Phi_{T}$ & 210 \\
\hline
\end{tabular}

Table 1: $\omega_{a b}$ is a second-rank traceless symmetric tensor; $\Phi_{a b c d}$ is a fourth-rank antisymmetric tensor, and the indices $1 \ldots 6$ correspond to $S O(6) \sim S U(4)_{P S}$, whereas $7 \ldots 0$ correspond to $S O(4) \sim S U(2)_{L} \otimes S U(2)_{R}$

TABLE II

\begin{tabular}{|c|c|c|}
\hline$G^{\prime}$ & $M_{X} / 10^{15} \mathrm{GeV}$ & $M_{R} / 10^{11} \mathrm{GeV}$ \\
\hline$S U(4)_{P S} \otimes S U(2)_{L} \otimes S U(2)_{R} \times D$ & $0.55 \cdot 1.6^{0 \pm 1}$ & $340 \cdot 1.3^{0 \pm 1}$ \\
\hline$S U(4)_{P S} \otimes S U(2)_{L} \otimes S U(2)_{R}$ & $5.3 \cdot 1.9^{0 \pm 1}$ & $1.4 \cdot 2.1^{0 \pm 1}$ \\
\hline$S U(3)_{c} \otimes S U(2)_{L} \otimes S U(2)_{R} \otimes U(1)_{B-L} \times D$ & $1.6 \cdot 2.8^{0 \pm 1}$ & $0.32 \cdot 1.8^{0 \pm 1}$ \\
\hline$S U(3)_{c} \otimes S U(2)_{L} \otimes S U(2)_{R} \otimes U(1)_{B-L}$ & $11 \cdot 2.1^{0 \pm 1}$ & $0.027 \cdot 3.3^{0 \pm 1}$ \\
\hline
\end{tabular}

Table 2: Values of $M_{X}$ and $M_{R}$ for different $G^{\prime}$ within the ESH. Being unable to evaluate the errors at two-loops, here we quote the errors obtained at one-loop; however, since the one-loop gives the main contribution to RGE, we expect about the same order of magnitude for the errors at one- and two-loops. 
The values of the scales $M_{X}$ and $M_{R}$ are reported in table II for the different models within the Extended Survival Hypothesis (ESH) (Barbieri, Morchio, Nanopoulos, and Strocchi, 1980), namely allowing only the scalars needed for breaking the symmetry at the lower scales to contribute to the RGE, and using the two-loop approximation for the RGE.

As one can see in table II, both models without D symmetry give sufficiently high values for the scale $M_{X}$, but the one with $G^{\prime} \supset S U(4)_{P S}$ predicts $M_{R} \sim 10^{11} \mathrm{GeV}$, whereas the one with $G^{\prime} \supset S U(3)_{c} \otimes U(1)_{B-L}$ leads to a value of $M_{R}$ of about two orders of magnitude smaller.

At one-loop one finds an upper limit for $M_{R}$,

$$
M_{R} \leq M_{Z} \exp \frac{\pi}{6 \alpha\left(M_{Z}\right)}\left[\frac{3}{2}-3 \sin ^{2} \theta_{W}\left(M_{Z}\right)-\frac{\alpha}{\alpha_{S}}\left(M_{Z}\right)\right],
$$

where the equality holds only for the model with $G^{\prime} \supset S U(4)_{P S} \times D$, whose prediction for $M_{X}$ is too small.

The ESH may be too drastic since in the 210 and 126 representations of $S O(10)$ there are multiplets with high quantum numbers, which may give important contributions to the RGE beyond the ones implied by symmetry, the only ones allowed within the ESH. This observation motivated Dixit and Sher to claim that huge uncertainties are introduced in the $S O(10)$-predictions if the ESH is removed (Dixit and Sher, 1989). However, as explicitly shown in the following, the mass spectrum of the scalars contributing to the RGE depends on the coefficients of the non-trivial invariants which appear in the scalar potential. These are constrained by demanding that the absolute minimum of the potential is in the direction giving the desired symmetry breaking pattern of the unified model considered. This will allow us to deduce rather restrictive conditions on the contributions of the scalars to RGE.

In this paper, we study the predictions for the scales $M_{X}$ and $M_{R}$ obtained under the more general hypothesis of allowing all the scalars to contribute to the RGE between the two highest scales, with their spectrum obeying the conditions necessary for obtaining the symmetry breaking pattern characterizing each model. By requiring for $M_{X}$ a value sufficiently high to comply with the lower limit on $\tau_{p \rightarrow e^{+} \pi^{0}}$ we shall find upper limits on $M_{R}$, which correspond to lower limits on the masses of the (almost) left-handed neutrinos. 


\section{Symmetry Breaking for Various Higgs Potentials}

$$
\text { A. } G^{\prime} \equiv S U(4)_{P S} \otimes S U(2)_{L} \otimes S U(2)_{R} \times D
$$

To construct a potential which gets its absolute minimum in the direction corresponding to the desired symmetry breaking pattern, one can use non-trivial positive definite invariants vanishing in that direction (Buccella and Ruegg, 1982; Kaymackcalan, Michel, Wali, Mc GLinn, and O'Raifeartaigh, 1986; Burzlaff and O'Raifeartaigh, 1990). In order to have a renormalizable potential the degree of these invariants should not exceed four.

In the case of the representation 54 (Shafi and Wetterich, 1979; Buccella, Cocco, and Wetterich, 1984) (whose generic element we denote by $\omega$ ) there is only one non-trivial quartic invariant,

$$
\left\|(\omega \omega)_{54}\right\|=\sum_{i}(\omega \omega)_{i}(\omega \omega)_{i}
$$

$\left((\omega \omega)_{i} \equiv \sum_{j, k} C_{j}^{54}{ }_{k}^{54}{ }_{i}^{54} \omega_{j} \omega_{k}, \quad(i, j, k=1 \ldots 54)\right.$ and the $C$ 's are $S O(10)$ Clebsh-Gordan $)$ which vanishes in the $S O(5) \otimes S O(5)$ direction, and is maximum in the $S O(9)$-invariant one. To get the absolute minimum in the $G_{422} \times D$-invariant direction $\omega_{L}$ one may consider the invariant

$$
\left\|(\omega \omega)_{54}+\alpha<\omega_{L}>\omega\right\|
$$

$\left(<\omega_{L}>\right.$ is the VEV of $\left.\omega_{L}\right)$, vanishing in the $\omega_{L}$ direction for $|\alpha|=\frac{1}{2 \sqrt{42}}$ since

$$
C_{\omega_{L}}^{54}{ }_{\omega_{L}}^{54} \underset{\omega}{\omega}=-\frac{1}{2 \sqrt{42}} \delta_{\omega_{L} \omega}
$$

The invariant defined in eq. (6) gets its absolute minimum in the $\omega_{L}$ direction (Abud, Buccella, Della Selva, Sciarrino, Fiore, and Immirzi, 1986) for $|\alpha|$ in an open range around $\frac{1}{2 \sqrt{42}}$ with the lower and upper limits corresponding to the degeneracy with the $S O(5) \otimes S O(5)$ or $S O(7) \otimes S O(3)$-invariant directions respectively. This implies a rather narrow range for the ratio of the two masses $\mathrm{m}(1,3,3)$ and $\mathrm{m}(20,1,1)$ of the scalars of the 54 representation (Buccella and Rosa, 1992):

$$
1.20<\frac{m(1,3,3)}{m(20,1,1)}<1.35
$$


Concerning the representation 126, a potential minimum in the $\mathrm{SU}(5)$-invariant direction $\psi_{0}$, which is also the only $G$-invariant in the 126, can be obtained (Buccella, Cocco, Sciarrino, and Tuzi, 1986) if the non-trivial part of the potential is given by $\left(\psi_{+}\right.$and $\psi_{-}$denote the 126 and $\overline{126}$ part respectively)

$$
\begin{aligned}
V_{\psi}\left(\psi_{+}, \psi_{-}\right) & =e_{1}\left\|\cos \theta_{1}\left(\psi_{+} \psi_{+}\right)_{4125}+\sin \theta_{1} e^{i \eta_{1}}\left(\psi_{-} \psi_{-}\right)_{4125}\right\|+e_{2}\left\|\left(\psi_{+} \psi_{+}\right)_{1050}\right\| \\
& +e_{3}\left\|\cos \theta_{2}\left(\psi_{+} \psi_{+}\right)_{54}+\sin \theta_{2} e^{i \eta_{2}}\left(\psi_{-} \psi_{-}\right)_{54}\right\|
\end{aligned}
$$

where

$$
\left(\psi_{+\beta} \psi_{+\beta}\right)_{n_{i}}=C_{\beta}^{126}{ }_{\beta}^{126}{ }_{i}^{n}<\psi_{+}>^{2},
$$

with $\beta$ defining the vector $\psi_{+}$in the 126 representation.

The expression given in eq. (9) is the most general combination of the non-trivial invariants with the only limitation that the moduli of the coefficients of $\left(\psi_{+} \psi_{+} \psi_{+} \psi_{+}\right)_{1}$ and of its Hermitean conjugate do not exceed an appropriate combination of $\left|e_{1}\right|$ and $\left|e_{3}\right|$.

The positive-definite invariants appearing in eq. (9) vanish in the $\mathrm{SU}(5)$-invariant direction $\psi_{0}$ since

$$
C_{\psi_{0}}^{126} \underset{\psi_{0}}{126} \underset{i}{r} \sim \delta_{r, 2695}
$$

Clearly, if $e_{i} \geq 0 V_{\psi}$ has its absolute minimum in the $\mathrm{SU}(5)$-invariant direction.

In any case, these considerations are rather academic since the direction of $\psi_{ \pm}$is determined mainly by the non-trivial invariants containing also $\omega$, which has a larger expectation value in the scheme we are interested in. By considering the tensor products

$$
\begin{aligned}
(54 \otimes 54)_{s} & =1 \oplus 54 \oplus 660 \oplus 770 \\
(126 \otimes 126)_{s} & =54 \oplus 1050 \oplus 2772 \oplus 4125 \\
126 \otimes \overline{126} & =1 \oplus 45 \oplus 210 \oplus 770 \oplus 5940 \oplus 8910
\end{aligned}
$$

(the subscript $\mathrm{s}$ means that one is considering the symmetric part of the product of two identical representations) we observe that there is one non-trivial invariant for the products $\psi_{+} \psi_{-}(\omega \omega)_{s},\left(\psi_{+} \psi_{+}\right)_{s}(\omega \omega)_{s}$ and $\left(\psi_{+} \psi_{+}\right)_{s} \omega$, which we can write respectively as

$$
\left\|\left(\psi_{+} \omega\right)_{\overline{126}}\right\|, \quad\left(\psi_{+} \psi_{+}\right)_{54} \times(\omega \omega)_{54} \text { and }\left(\psi_{+} \psi_{+}\right)_{54} \times \omega .
$$


All the invariants in (13) vanish in the direction $\omega_{L}, \psi_{0}$ since the 54 does not contains $\mathrm{SU}(5)$ singlets; hence

$$
\left(\psi_{+0} \psi_{+0}\right)_{54}=0
$$

and

$$
C_{\psi_{0}}^{126} \underset{\omega_{L}}{54} \bar{i} \bar{i}=0
$$

since $\psi_{0} \omega_{L}$ is the $G$-singlet of a 24 of $\mathrm{SU}(5)$ and the only $G$-singlet of the $\overline{126}$ is a singlet also of $\mathrm{SU}(5)$. Of the three invariants defined in eq. (13) the first one is positive-definite while the other two change sign under the phase transformation $\psi_{+} \rightarrow i \psi_{+}$. Thus, to have the minimum in the desired direction it is necessary to take a positive value for the coefficient of the first invariant and to have sufficiently small coefficients for the other two, which both tend to choose different directions for $\psi_{+}$, once $\omega$ is fixed in the $\omega_{L}$ direction.

\section{B. The 210 representation}

Because in all the other $\mathrm{SO}(10)$ models that we discuss the Higgs potential depends on the reducible representation which is the sum of the 210, 126, $\overline{126}$ and 10 (whose generic elements we indicate with $\Phi, \psi_{+}, \psi_{-}, \rho$ respectively), we give a general analysis of the orbit structure of this potential, which we indicate with $\mathrm{V}\left(\Phi, \psi_{+}, \psi_{-}, \rho\right)$ and can be written (Abud, Buccella, Rosa, and Sciarrino, 1989) as

$$
\begin{aligned}
V\left(\Phi, \psi_{+}, \psi_{-}, \rho\right) & =V_{0}(\|\Phi\|,\|\psi\|,\|\rho\|)+V_{\Phi}(\Phi)+V_{\psi}\left(\psi_{+}, \psi_{-}\right) \\
& +V_{\Phi, \psi}\left(\Phi, \psi_{+}, \psi_{-}\right)+V_{\Phi, \psi, \rho}\left(\Phi, \psi_{+}, \psi_{-}, \rho\right)
\end{aligned}
$$

where $V_{0}$ is a function of the fields' norms only, hence it is isotropic in the space of representations and does not affect the direction of the potential minimum.

The final little group is obtained from the intersection of each of the little groups of the irreducible components of the $210 \oplus 126 \oplus \overline{126} \oplus 10 . V_{\Phi}(\Phi)$ breaks $S O(10)$ to $G^{\prime}$, $V_{\psi}\left(\psi_{+}, \psi_{-}\right)+V_{\Phi, \psi}\left(\Phi, \psi_{+}, \psi_{-}\right)$realizes the second symmetry breaking step to $G$ and finally $V_{\Phi, \psi, \rho}\left(\Phi, \psi_{+}, \psi_{-}, \rho\right)$ breaks down to $S U(3) \otimes U(1)$.

Let $r, s$ and $t$ be the fields' norms in the vacuum state:

$$
r=\sqrt{\left\|\Phi_{0}\right\|}, \quad s=\sqrt{\left\|\psi_{0}\right\|}, \quad t=\sqrt{\left\|\rho_{0}\right\|} ;
$$


we can then write

$$
\begin{aligned}
V_{0}(\|\Phi\|,\|\psi\|,\|\rho\|) & =h_{\Phi \Phi}\left[\|\Phi\|-r^{2}\right]^{2}+h_{\psi \psi}\left[\|\psi\|-s^{2}\right]^{2}+h_{\rho \rho}\left[\|\rho\|-t^{2}\right]^{2} \\
& +h_{\Phi \psi}\left[\|\Phi\|-r^{2}\right]\left[\|\psi\|-s^{2}\right]+h_{\Phi \rho}\left[\|\Phi\|-r^{2}\right]\left[\|\rho\|-t^{2}\right] \\
& +h_{\psi \rho}\left[\|\psi\|-s^{2}\right]\left[\|\rho\|-t^{2}\right] .
\end{aligned}
$$

Choosing $h$ so that we have a positive-definite quadratic form, $V_{0}$ is minimum for

$$
\|\Phi\|=r^{2}, \quad\|\psi\|=s^{2}, \quad\|\rho\|=t^{2} .
$$

The remaining terms are $\left(V_{\psi}\right.$ is defined in eq. (9)) (Buccella, 1988):

$$
\begin{aligned}
V_{\Phi}(\Phi)= & A\left\|(\Phi \Phi)_{45}\right\|+B\left\|(\Phi \Phi)_{54}\right\|+C\left\|(\Phi \Phi)_{210}\right\| \\
& -r D\left[(\Phi \Phi)_{210} \times \Phi\right]_{1}, \\
V_{\Phi, \psi}\left(\Phi, \psi_{+}, \psi_{-}\right)= & f_{1}\left\|\cos \theta_{3}\left(\Phi \psi_{+}\right)_{10}+\sin \theta_{3} e^{i \eta_{3}}\left(\Phi \psi_{-}\right)_{10}\right\| \\
+ & f_{2}\left\|\cos \theta_{4}\left(\Phi \psi_{+}\right)_{120}+\sin \theta_{4} e^{i \eta_{4}}\left(\Phi \psi_{-}\right)_{120}\right\| \\
+ & f_{3}\left\|\cos \theta_{5}\left(\Phi \psi_{+}\right)_{320}+\sin \theta_{5} e^{i \eta_{5}}\left(\Phi \psi_{-}\right)_{320}\right\| \\
+ & f_{4}\left\|\left(\Phi \psi_{+}\right)_{126}+k \psi_{+}\right\|+f_{5}\left[\left(\psi_{+} \psi_{-}\right)_{45} \times(\Phi \Phi)_{45}\right]_{1}, \\
& \\
V_{\Phi, \psi, \rho}\left(\Phi, \psi_{+}, \psi_{-}, \rho\right)= & {\left[P_{10}\left(\Phi, \psi_{+}, \psi_{-}\right) \times \rho\right]_{1} } \\
+ & {\left[\left(q_{1}(\Phi \Phi)_{54}+q_{2}\left(\psi_{+} \psi_{+}\right)_{54}+q_{2}^{*}\left(\psi_{-} \psi_{-}\right)_{54}\right) \otimes(\rho \rho)_{54}\right]_{1} . }
\end{aligned}
$$

$(\Phi \Phi)_{d}$ is the representation of dimension $d$ in the product $210 \otimes 210$, more precisely $(\Phi \Phi)_{d} \equiv \sum_{i} C_{\Phi}^{210} \underset{\Phi}{210}{ }_{i}^{d} d_{i}$; analogously for $(\psi \psi),(\Phi \psi)$ and $(\rho \rho) . P_{10}$ is the most general third order polynomial which transforms like a 10.

The expression of $V_{\Phi, \psi}$ given in eq. (21) contains all non-trivial invariants that can be built with $\Phi$ and $\psi_{ \pm}$, with the only limitation that the coefficients of the invariants $\left(\Phi \psi_{ \pm}\right)_{l} \times\left(\Phi \psi_{ \pm}\right)_{l} \quad(l=10,120,320)$ have modulus sufficiently small with respect to the coefficients of $\left\|(\Phi \psi)_{l}\right\|$. 
The symmetry breaking pattern of the models that we analyze only differ in the first step (they have different $G^{\prime}$ ). We can therefore start by giving a general discussion of the terms in the Higgs potential which are responsible for the second and third symmetry breaking step. $V_{\psi}$, as defined in eq. (9), gets certainly its minimum in the $\mathrm{SU}(5)$-invariant direction $\psi_{0}$ if the $e_{i}$ 's are all positive. As far as $V_{\Phi, \psi}$ is concerned, if $\Phi$ and $\psi$ admit a common little group $G$ then the terms $(\Phi \psi)_{r}$ with $r \equiv 10,120,320$ vanish because these representations do not contain singlets under $G$ and therefore the first three terms in eq. (21) vanish in the $G$ direction.

The inhomogeneous term $\left\|\left(\Phi \psi_{+}\right)_{126}+k \psi_{+}\right\|$will vanish in the same direction if we take $\Phi$ in a direction $\Phi_{0}$ belonging to the three-dimensional stratum invariant under $G$ and

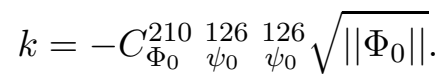

In fact, taking $\Phi$ and $\psi$ singlets of $G$ the only non-vanishing component of the product $\Phi \psi_{+}$is in the direction $\psi_{0}$. In conclusion, taking $f_{5}$ small enough, $V_{\Phi, \psi}$ in eq. (21) will be minimum in the desired direction. To simplify our discussion we take $f_{5}=0$.

Let us finally consider $V_{\Phi, \psi, \rho}$. Since, as we said, the 10 representation does not contain singlets under $G, P_{10}$ vanishes at $\Phi=\Phi_{0}, \psi=\psi_{0}$. Since the 54 does not contain $\operatorname{SU}(5)$ singlets, $\left(\psi_{+} \psi_{+}\right)_{54}$ and $\left(\psi_{-} \psi_{-}\right)_{54}$ vanish in the $\mathrm{SU}(5)$-invariant direction. We have to choose the sign of $q_{1}$ in such a way that $q_{1}\left(\Phi_{0} \Phi_{0}\right)_{54} \times(\rho \rho)_{54}$ is smaller in the $(1,2,2) \rho$-direction (with respect to $\left.G_{422}\right)$ rather than in the $(6,1,1)$; with our conventions for $S O(10)$ Clebsh-Gordan coefficients this happens if

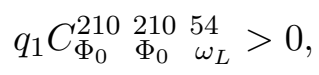

where $\Phi_{0}$ is the direction of the $210 \mathrm{VEV}$ and $\omega_{L}$ is the $G_{422}$-invariant direction introduced during the discussion of the breaking with the 54 and obeying eq. (7).

In conclusion, allowing for $V_{\Phi}(\Phi)$ being minimum in a direction with little group $G^{\prime}$ containing $G$, we achieve our goal.

In the 210 representation there are three independent $G$-singlets; therefore, the most general $G$-invariant direction lies on the three-dimensional vector space (Abud, Buccella, 
Rosa, and Sciarrino, 1989)

$$
\begin{aligned}
\Phi_{0}\left(z_{1}, z_{2}, z_{3}\right) & =z_{1} \frac{\Phi_{1234}+\Phi_{1256}+\Phi_{3456}}{\sqrt{3}} \\
& +z_{2} \frac{\Phi_{1278}+\Phi_{1290}+\Phi_{3478}+\Phi_{3490}+\Phi_{5678}+\Phi_{5690}}{\sqrt{6}} \\
& +z_{3} \Phi_{7890} \equiv z_{1} \Phi_{L}+z_{2} \hat{\Phi}+z_{3} \Phi_{T},
\end{aligned}
$$

with $z_{1}^{2}+z_{2}^{2}+z_{3}^{2}=1$. For particular values of $z_{i}, \Phi_{0}$ gets the following stability groups:

$$
\begin{aligned}
& z_{1} / \sqrt{3}=z_{2} / \sqrt{6}=z_{3} \quad \longrightarrow \quad S U(5) \otimes U(1) \\
& z_{1}=z_{2}=0 \quad \longrightarrow \quad S U(4)_{P S} \otimes S U(2)_{L} \otimes S U(2)_{R} \\
& z_{2}=z_{3}=0 \quad \longrightarrow \quad S U(3)_{C} \otimes S U(2)_{L} \otimes S U(2)_{R} \otimes U(1)_{B-L} \times D \\
& z_{2}=0,\left|z_{1}\right| \neq 0,1 \quad \longrightarrow \quad S U(3)_{C} \otimes S U(2)_{L} \otimes S U(2)_{R} \otimes U(1)_{B-L} \\
& \forall z_{i} \quad G^{\prime} \supset S U(3)_{C} \otimes S U(2)_{L} \otimes U(1)_{T_{3 R}} \otimes U(1)_{B-L} .
\end{aligned}
$$

It is possible to show that in the 210 there are four non-trivial independent invariants with degree $\leq 4$ which can be written as done in eq. (20). For the phenomenological reasons mentioned in the introduction, we are interested in intermediate symmetries containing $S U(2)_{R}$ and (or) $S U(4)_{P S}$, therefore we look for the minimum of $V_{\Phi}(\Phi)$ in the 2- dimensional stratum characterized by the condition $z_{2}=0$. Writing the expression of $V_{\Phi}$ in this stratum $\left(\Phi_{0} \equiv z_{1} \Phi_{L}+z_{3} \Phi_{T}\right)$ we get

$$
\begin{aligned}
V_{2-\operatorname{dim} .}\left(z_{1}, z_{3}\right) \equiv \frac{V_{\Phi}\left(\Phi_{0}\left(z_{1}, 0, z_{3}\right)\right)}{r^{4}} & =\frac{2}{35} A\left(z_{1} z_{3}\right)^{2}+\frac{1}{210} B\left(2 z_{1}^{2}-3 z_{3}^{2}\right)^{2} \\
& +\frac{2}{135} C z_{1}^{4}-\frac{2}{3 \sqrt{30}} D z_{1}^{3} .
\end{aligned}
$$

Because $z_{1}^{2}+z_{3}^{2}=1$ and using the new parameters

$$
\alpha=\frac{4}{945}(-108 A+225 B+28 C), \quad \beta=\frac{2}{\sqrt{30}} D, \quad \gamma=\frac{4}{35}(2 A-5 B), \quad \delta=\frac{3}{70} B
$$

for later convenience, eq. (26) becomes

$$
V_{2-\text { dim. }}=\frac{\alpha}{8} z_{1}^{4}-\frac{\beta}{3} z_{1}^{3}+\frac{\gamma}{4} z_{1}^{2}+\delta .
$$


To have a relative minimum at $\left.\tilde{z}_{1} \in\right]-1,1[$ we must impose the conditions

$$
\left(\frac{d V_{2-d i m .}}{d z_{1}}\right)_{\tilde{z}_{1}}=0, \quad\left(\frac{d^{2} V_{2-d i m}}{d z_{1}^{2}}\right)_{\tilde{z}_{1}}>0 .
$$

The first condition is automatically satisfied at $z_{1}=0$ corresponding to the critical orbit with little group $G_{422}$.

Moreover, we should have a positive-definite Hessian

$$
\left.\frac{\partial^{2} V_{\Phi}(\Phi)}{\partial \Phi_{i} \partial \Phi_{j}}\right|_{\Phi_{0}\left(\tilde{z}_{1}\right)}>0
$$

in the subspace subtended by the scalars of the 210 surviving the Higgs mechanism. The values of these masses, as a function of the parameters defined in eq. (27) and of $z_{1}$ and $z_{3}$, are reported in the Appendix, where one finds also the masses of the $126 \oplus \overline{126}$ depending on the $f_{i}$ 's defined in eq. (21).

Finally, we must be sure that $V_{2-\operatorname{dim}}\left(\tilde{z}_{1}\right)$ is an absolute minimum in the 3-dimensional stratum $\Phi_{0}\left(z_{1}, z_{2}, z_{3}\right)$, i.e.

$$
V_{3-\operatorname{dim} .}\left(z_{1}, z_{2}, z_{3}\right) \equiv \frac{V_{\Phi}\left(\Phi_{0}\left(z_{1}, z_{2}, z_{3}\right)\right)}{r^{4}}>V_{2-\operatorname{dim} .}\left(\tilde{z}_{1}\right) \quad \forall\left(z_{1}, z_{2}, z_{3}\right) \neq\left(\tilde{z}_{1}, 0, \pm \sqrt{1-\tilde{z}_{1}^{2}}\right) .
$$

An indefinite metric for the Hessian would imply that $\Phi_{0}\left(z_{i}\right)$ is not an absolute minimum; hence the study of its positivity is a way to explore the existence of $V(\Phi)<V_{2-\operatorname{dim}}$. $\left(\tilde{z}_{1}\right)$ in directions not belonging to the $S U(3) \otimes S U(2) \otimes S U(2) \otimes U(1) \equiv G_{3221}$-invariant stratum.

Using the following expression for $V_{3-\operatorname{dim} .}\left(z_{1}, z_{2}, z_{3}\right)$,

$$
\begin{aligned}
& V_{3-\operatorname{dim} .}\left(z_{1}, z_{2}, z_{3}\right)=\frac{\alpha}{8} f_{\alpha}\left(z_{i}\right)-\frac{\beta}{3} f_{\beta}\left(z_{i}\right)+\frac{\gamma}{4} f_{\gamma}\left(z_{i}\right)+\frac{\delta}{9} f_{\delta}\left(z_{i}\right), \\
f_{\alpha}\left(z_{i}\right)= & \left(z_{1}^{2}+z_{2}^{2}\right)^{2}+z_{2}^{2}\left(2 z_{1}+\sqrt{z_{3}}\right)^{2}+\frac{3}{4} z_{2}^{4} \\
f_{\beta}\left(z_{i}\right)= & z_{1}^{3}+3 z_{1} z_{2}^{2}+\sqrt{27 / 4} z_{2}^{2} z_{3}, \\
f_{\gamma}\left(z_{i}\right)= & \left(z_{1} z_{3}+z_{2}^{2} / \sqrt{3}\right)^{2}+z_{1}^{2} z_{2}^{2}+\left(z_{1}^{2}+z_{2}^{2}\right)^{2}+z_{2}^{2}\left(2 z_{1}+\sqrt{3} z_{3}\right)^{2}+\frac{3}{4} z_{2}^{4}, \\
f_{\delta}\left(z_{i}\right)= & 30\left(z_{1} z_{3}+z_{2}^{2} / \sqrt{3}\right)^{2}+30 z_{1}^{2} z_{2}^{2}+\left(2 z_{1}^{2}-z_{2}^{2} / 2-3 z_{3}^{2}\right)^{2} \\
& +5\left(z_{1}^{2}+z_{2}^{2}\right)^{2}+5 z_{2}^{2}\left(2 z_{1}+\sqrt{3} z_{3}\right)^{2}+\frac{15}{4} z_{2}^{4},
\end{aligned}
$$


Figure 2: $\quad I_{0}=\left\{\left(z_{1}, z_{2}\right): f_{\delta}\left(z_{1}, z_{2}\right)=0\right\}, \quad I_{+}=\left\{\left(z_{1}, z_{2}\right): f_{\delta}\left(z_{1}, z_{2}\right)>0\right\}, \quad I_{-}=$ $\left\{\left(z_{1}, z_{2}\right): f_{\delta}\left(z_{1}, z_{2}\right)<0\right\}$

eq. (31) becomes $\left(z_{3}=\sqrt{1-z_{1}^{2}-z_{2}^{2}}\right)$

$$
f_{\delta}\left(z_{1}, z_{2}\right) \delta>f_{\alpha}\left(z_{1}, z_{2}\right) \alpha+f_{\beta}\left(z_{1}, z_{2}\right) \beta+f_{\gamma}\left(z_{1}, z_{2}\right) \gamma+\sigma
$$

where $\sigma$ is a constant depending on $\tilde{z}_{1}$ and hence on the residual symmetry of the model. We distinguish the three regions represented in fig.2.

In order to satisfy the inequality (37) in each of the three regions one needs that

$$
f_{\alpha} \alpha+f_{\beta} \beta+f_{\gamma} \gamma+\sigma<0, \quad \delta_{+}<\delta<\delta_{-},
$$

with

$$
\begin{aligned}
& \delta_{+} \equiv \max \left\{\frac{\left[\alpha f_{\alpha}\left(z_{i}\right)+\beta f_{\beta}\left(z_{i}\right)+\gamma f_{\gamma}\left(z_{i}\right)+\sigma\right]}{f_{\delta}\left(z_{i}\right)}\right\}_{\left\{\left(z_{1}, z_{2}, z_{3}\right): f_{\delta}\left(z_{i}\right)>0\right\}} \\
& \delta_{-} \equiv \min \left\{\frac{\left[\alpha f_{\alpha}\left(z_{i}\right)+\beta f_{\beta}\left(z_{i}\right)+\gamma f_{\gamma}\left(z_{i}\right)+\sigma\right]}{f_{\delta}\left(z_{i}\right)}\right\}_{\left\{\left(z_{1}, z_{2}, z_{3}\right): f_{\delta}\left(z_{i}\right)<0\right\}} .
\end{aligned}
$$

By studying the last equation, combined with eq. (30), we find that it is sufficient to verify that the minimum obtained is lower than the value corresponding to the direction invariant under the maximal little group $S U(5) \otimes U(1)$, which lies in the $I_{+}$region.

1. $G^{\prime} \equiv S U(4)_{P S} \otimes S U(2)_{L} \otimes S U(2)_{R}$

In Buccella, Cocco, Sciarrino, and Tuzi (1986) one has considered a potential $V_{\Phi}$ with positive coefficients for the non-trivial invariants $\left\|(\Phi \Phi)_{45}\right\|,\left\|(\Phi \Phi)_{210}\right\|$ and $\left\|(\Phi \Phi)_{1050}\right\|$, which vanish 
in the $G_{422}$-invariant direction. If the cubic term $(\Phi \Phi)_{210} \times \Phi$ is absent or its coefficient is sufficiently small, the absolute minimum of $V_{\Phi}$ is in the $G_{422}$-invariant direction. In any case, a necessary condition to have a minimum in the desired direction is

$$
V\left(\Phi_{T}\right)<V\left(\Phi_{L}\right)
$$

which implies the following constraint on the mass spectrum:

$$
\frac{2-\sqrt{3}}{2+\sqrt{3}}<\frac{m^{2}(15,3,1)}{m^{2}(15,1,3)}<\frac{2+\sqrt{3}}{2-\sqrt{3}}
$$

From the identity

$$
\left\|(\Phi \Phi)_{54}\right\|=-\frac{35}{14}\left\|(\Phi \Phi)_{45}\right\|-\frac{3}{7}\left\|(\Phi \Phi)_{1050}\right\|+\frac{15}{28}\left\|(\Phi \Phi)_{210}\right\|+\frac{1}{10}\|\Phi\|^{2}
$$

it is straightforward to translate the positivity of the coefficients of $\left\|(\Phi \Phi)_{r}\right\|(\mathrm{r}=45,210,1050)$ into the following inequalities for the parameters defined in eq. (27)

$$
\gamma>0, \quad-\frac{27}{160}(\alpha+2 \gamma)<\delta<0
$$

while $\beta=0$ corresponds to the vanishing of the coefficient of the cubic term. If $\beta=0$ and the inequalities (44) old, the absolute minimum of $V_{\Phi}$ is in the $G_{422}$-invariant direction $\Phi_{T}$. The necessary condition corresponding to the inequality (41) reads

$$
|\beta|<\frac{3}{8}(\alpha+2 \gamma)
$$

From eqs. (29), (30) and (38) we find the following necessary conditions to get the absolute minimum in the $G_{422}$-invariant direction

$$
\gamma>0, \quad \delta_{T}<\delta<0, \quad \begin{cases}-2 \gamma<\alpha \leq 2 \gamma & 0 \leq|\beta|<\frac{3}{8}(\alpha+2 \gamma) \\ \alpha>2 \gamma & 0 \leq|\beta|<\frac{3}{2 \sqrt{2}} \sqrt{\alpha \gamma}\end{cases}
$$

where:

$$
\delta_{T} \equiv \frac{1}{32 \cdot 15}(-81 \alpha+24 \sqrt{30} \beta-7 \cdot 27 \gamma)
$$


2. $G^{\prime} \equiv S U(3)_{c} \otimes S U(2)_{L} \otimes S U(2)_{R} \otimes U(1)_{B-L} \times D$

Two positive-definite invariants vanish in the $\Phi_{L}$ direction (Buccella and Rosa, 1987), i.e.

$$
\left\|(\Phi \Phi)_{45}\right\| \text { and }\left\|(\Phi \Phi)_{210}-C_{\Phi_{L}}^{210} \stackrel{\Phi}{L}_{L}^{210} \Phi_{L}^{210}<\Phi>\Phi\right\|
$$

In fact, from the rule

$$
C_{a b c d \text { efgh }}^{210} \underset{\text { il }}{210}=\frac{1}{\sqrt{70}} \epsilon_{a b c d e f g h i l}
$$

it follows that

$$
\left(\Phi_{L} \Phi_{L}\right)_{45}=0
$$

and $\Phi_{L}$ enjoys the property of any critical direction

$$
C_{\Phi_{L}}^{210} \underset{\Phi_{L}}{210} \Phi_{i}^{210} \sim \delta_{\Phi_{L} \Phi_{i}}
$$

which implies a vanishing value for the second invariant defined by eq. (48).

Because other critical directions $\Phi$, invariant under $G_{422}$ or $S U(5)$ or $G(2) \otimes S U(2)$ (Baseq, Meljanac, and O'Raifeartaigh, 1989) give rise to different values for $C_{\Phi}^{210} \underset{\Phi}{210} \underset{\Phi}{210}$ the conclusion is that the minimum of the potential is in the $G_{3221} \times D$-invariant direction with positive coefficients for this two invariants and smaller coefficients for the others. With our parametrization the corresponding region is

$$
\gamma>0, \quad \beta>0, \quad-\frac{3}{40} \gamma<\delta<0, \quad\{|\delta|,|9 \alpha+18 \gamma-12 \beta+40 \delta|\} \text { "small enough". }
$$

Again from eqs. (29), (30) and (38) we find that the following are the necessary conditions to get the absolute minimum in the direction $\Phi_{L}$ :

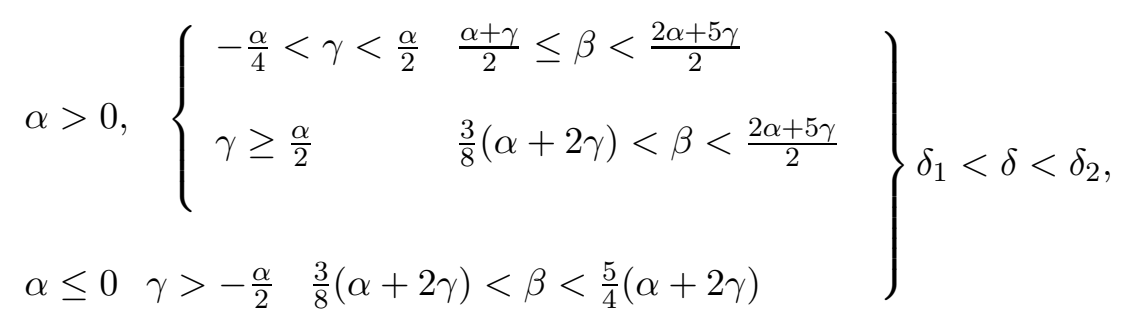


where

$$
\begin{aligned}
\delta_{1} & \equiv \max \left\{\frac{-5 \alpha+4 \beta-10 \gamma}{20}, \frac{9\left(-\alpha+\beta-\frac{5}{2} \gamma\right)}{80}, \frac{-51 \alpha+8(3 \sqrt{30}-10) \beta-129 \gamma}{32 \cdot 15}\right\} \\
\delta_{2} & \equiv \min \left\{0, \frac{1}{20}(-3 \alpha+6 \beta-5 \gamma)\right\}
\end{aligned}
$$

3. $G^{\prime} \equiv S U(3)_{c} \otimes S U(2)_{L} \otimes S U(2)_{R} \otimes U(1)_{B-L}$

The $G_{3221}$-invariant stratum can be described, as long as $\sin 2 \theta \neq 0$, by

$$
\Phi(\theta)=\cos \theta \Phi_{L}+\sin \theta \Phi_{T}
$$

Abud et al. (Abud, Buccella, Rosa, and Sciarrino, 1989) considered the symmetry breaking direction $\Phi\left(\theta^{*}\right)$ with $\theta^{*} \equiv \operatorname{arcos} \sqrt{\frac{3}{5}}$. By exploiting the fact that $\left\|\left[\Phi\left(\theta^{*}\right) \Phi(\theta *)\right]_{54}\right\|=0$, they found sufficient conditions to get $G^{\prime}=G_{3221}$. However, as already pointed out in Abud, Buccella, Rosa, and Sciarrino (1989), the direction $\Phi\left(\theta^{*}\right)$ does not obey the inequality (24) necessary to construct a $V_{\Phi=\Phi_{0}, \psi, \rho}$ breaking $S U(2)_{L}$.

A search for different directions in the $\Phi(\theta)$-stratum has been performed in the theses of two of us (Amelino-Camelia, 1989; Pisanti, 1992). In particular, the challenging program of finding all the conditions on the potential parameters necessary to get the minimum in the $\Phi(\theta)$-stratum has been carried out in Pisanti (1992).

By imposing that $V_{\Phi}$, restricted on the 3-dimensional stratum defined by eq. (25), has its absolute minimum at $z_{2}=0$ and $z_{1} z_{3} \neq 0$, and by requiring the positivity of the mass spectrum, which gives some confidence in the fact that the absolute minimum of $V_{\Phi}$ does not lie in a direction different from the ones defined by eq. (56), we get four cases, all obeying the conditions

$$
\alpha>0, \quad \delta_{S U(5)}<\delta
$$

$\left(\delta_{S U(5)}\right.$ is the value of $\delta$ for which there is a degeneracy with the $S U(5) \otimes U(1)$-invariant direction), where

$$
\begin{aligned}
\delta_{S U(5)} & \equiv \frac{1}{32 \cdot 15}\left(-81 \alpha+24 \sqrt{30} \beta-7 \cdot 27 \gamma+\frac{10}{3} \sigma\right) \\
\sigma & \equiv-\frac{3}{\alpha^{3}}\left[8 \beta^{4}+3 \alpha^{2} \gamma^{2}-12 \alpha \beta^{2} \gamma+8 \beta\left(\beta^{2}-\alpha \gamma\right)^{\frac{3}{2}}\right]
\end{aligned}
$$


and with the further constraints on the parameters described in table III.

TABLE III

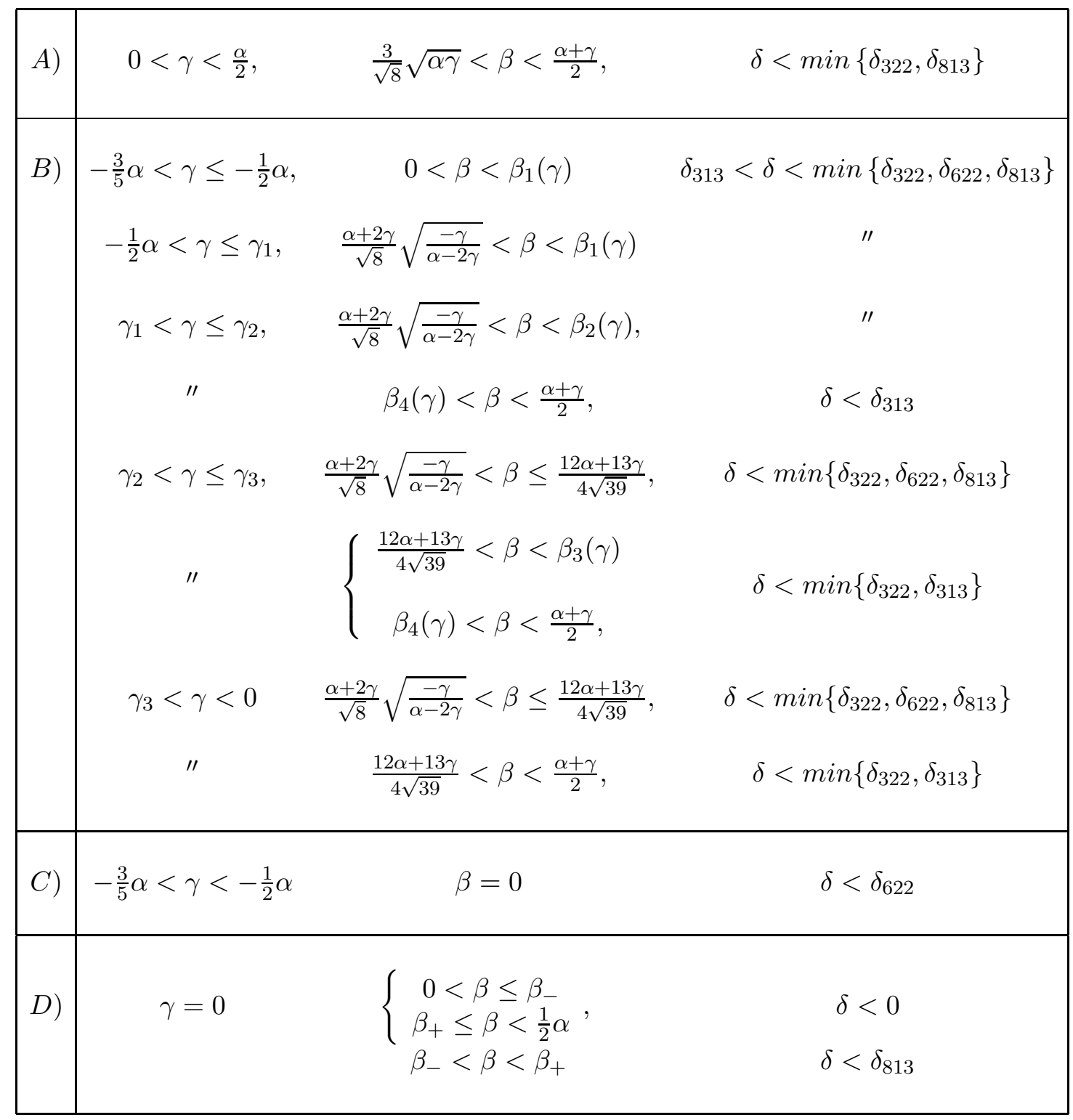

In table III, $\delta_{i}$ is the value of $\delta$ for which $m_{i}$ vanishes (e.g. $m(3,2,2,-2 / 3)=0$ for $\left.\delta=\delta_{322}\right)$; moreover: 
- $\gamma_{1}=\frac{-91+12 \sqrt{30}}{169-12 \sqrt{30}} \alpha \simeq-0.245 \alpha$

- $\gamma_{2}=-3 / 19 \alpha$

- $\beta_{3}(\gamma), \beta_{4}(\gamma)$ are defined by the equality $\delta_{313}\left(\beta_{3,4}, \gamma\right)=\delta_{S U(5)}\left(\beta_{3,4}, \gamma\right)$

- $\beta_{1}(\gamma)$ is such that $\delta_{313}\left(\beta_{1}, \gamma\right)=\delta_{813}\left(\beta_{1}, \gamma\right)$

- $\beta_{2}(\gamma)$ is such that $\delta_{313}\left(\beta_{2}, \gamma\right)=\min \left\{\delta_{322}\left(\beta_{2}, \gamma\right), \delta_{813}\left(\beta_{2}, \gamma\right)\right\}$

- $\gamma_{3}$ is defined by $\beta_{3}(\gamma)=\beta_{4}(\gamma)$

- $\delta_{322}=-\gamma / 10$

- $\delta_{622}=\frac{3\left[-2 \beta^{2} \gamma+2 \alpha \beta^{2}+\alpha \gamma^{2}+2 \beta(\alpha-\gamma) \sqrt{\beta^{2}-\alpha \gamma}\right]}{\left.10\left[3 \alpha^{2}-2 \beta^{2}+\gamma-2 \beta\right) \sqrt{\beta^{2}-\alpha \gamma}\right]}$

- $\delta_{313}=9 \frac{\frac{3}{4}(\alpha+2 \gamma)-\beta z_{1}-\sqrt{3} \beta z_{3}-\frac{9 \alpha+54 \gamma}{36} z_{1}^{2}+\frac{9 \alpha+21 \gamma}{\sqrt{108}} z_{1} z_{3}+\beta z_{1}^{3}-\frac{\alpha}{2} z_{1}^{4}}{20 z_{1}\left(z_{1}-2 \sqrt{3} z_{3}\right)}$

- $\delta_{813}=9 \frac{\frac{3}{4}(\alpha+2 \gamma)+\beta z_{1}-\sqrt{3} \beta z_{3}-\frac{27 \alpha+90 \gamma}{36} z_{1}^{2}-\frac{9 \alpha+21 \gamma}{\sqrt{108}} z_{1} z_{3}+\beta z_{1}^{3}-\frac{\alpha}{2} z_{1}^{4}}{40 z_{1}\left(z_{1}+\sqrt{3} z_{3}\right)}$

- $\beta_{ \pm}=\frac{\sqrt{27 \pm 12 \sqrt{2}}}{14} \alpha \simeq\left\{\begin{array}{c}0.4756 \alpha \\ 0.2262 \alpha\end{array}\right.$

\section{Renormalization-Group Equations}

In the following we study the implications of the RGE for each of the $\mathrm{SO}(10)$ models that we have considered. In the RGE-analysis presented in Mohapatra and Parida (1993) and Deshpande, Keith, and Palash Pal (1992) the simplifying ESH has been assumed. We shall remove this assumption, and consider all possible scalar contributions to the RGE above $M_{R}$ that are compatible with the necessary conditions which we obtained in the preceding 
section by demanding that the absolute minimum of the potential is in the direction giving the desired symmetry breaking pattern.

The evolution of the coupling constants $\alpha_{i}(\mu)$ is governed by the Gell-Mann Low equation

$$
\mu \frac{d}{d \mu} \alpha_{i}(\mu)=\beta_{i}\left(\alpha_{i}(\mu)\right)
$$

with

$$
\beta_{i}\left(\alpha_{i}(\mu)\right)=a_{i} \alpha_{i}^{2}(\mu)+\sum_{j} b_{i j} \alpha_{j}(\mu) \alpha_{i}^{2}(\mu)+\ldots
$$

(no sum on the repeated indices).

The general expression for $a_{i}$ and $b_{i j}$ for a gauge group $G=G_{1} \otimes G_{2}$ is (Jones, 1982)

$$
\begin{aligned}
a_{i} & =\frac{1}{2 \pi}\left[\frac{2}{3} \sum_{F} T\left(F_{i}\right) F_{j}+\frac{1}{6} \sum_{S} T\left(S_{i}\right) S_{j}-\frac{11}{3} C_{2}\left(G_{i}\right)\right] \quad(i=1,2) \\
b_{i j} & =\frac{1}{8 \pi^{2}}\left[2 \sum_{F} C_{2}\left(F_{j}\right) F_{j} T\left(F_{i}\right)+2 \sum_{S} C_{2}\left(S_{j}\right) S_{j} T\left(S_{i}\right)\right] \quad(i, j=1,2 ; i \neq j) \\
b_{i i} & =\frac{1}{8 \pi^{2}}\left[\sum_{F}\left(\frac{10}{3} C_{2}\left(G_{i}\right)+2 C_{2}\left(F_{i}\right)\right) F_{j} T\left(F_{i}\right)\right] \\
& +\frac{1}{8 \pi^{2}}\left[\sum_{S}\left(\frac{1}{3} C_{2}\left(G_{i}\right)+2 C_{2}\left(S_{i}\right)\right) S_{j} T\left(S_{i}\right)-\frac{34}{3}\left(C_{2}\left(G_{i}\right)\right)^{2}\right] \quad(i=1,2) .
\end{aligned}
$$

- $F_{i}$ and $S_{i},(i=1,2)$ are the dimension of the multiplet that classifies fermions and scalars respectively under $G_{1} \otimes G_{2}$.

- $C_{2}(F)$ is the second-order Casimir of the representation $\mathrm{F}$.

- $G_{i}$ is the adjoint representation.

- $T(F) \equiv \frac{C_{2}(F) F}{\operatorname{dim}(\text { adjoint })}$.

- If $G=U(1)$ with generator $\sigma$ then $C_{2}(G)=0, C_{2}(F)=\frac{1}{2} \operatorname{Tr}\left(\sigma(F)^{2}\right)=T(F)$.

At the scales $M_{I}$ of the spontaneous symmetry breaking we impose for the coupling constants, which for symmetry reasons are equal above $M_{I}$ whereas they evolve independently 
below $M_{I}$, the matching conditions (Weinberg, 1980; Mohapatra and Parida, 1993)

$$
\frac{1}{\alpha_{i}\left(M_{I}\right)}=\frac{1}{\alpha_{I}}-\frac{C_{2}\left(G_{i}\right)}{12 \pi} \text {. }
$$

A. $G^{\prime} \equiv S U(4) \otimes S U(2) \otimes S U(2) \times D$

In writing the RGE we define the two parameters $A$ and $B$ as

$$
\begin{aligned}
A & \equiv \frac{6 \pi}{11 \alpha\left(M_{Z}\right)}\left(\sin ^{2} \theta_{W}\left(M_{Z}\right)-\frac{\alpha}{\alpha_{S}}\left(M_{Z}\right)\right) \\
B & \equiv \frac{\pi}{11 \alpha\left(M_{Z}\right)}\left(\frac{3}{2}-3 \sin ^{2} \theta_{W}\left(M_{Z}\right)-\frac{\alpha}{\alpha_{S}}\left(M_{Z}\right)\right),
\end{aligned}
$$

where (Anselmo, Cifarelli, and Zichichi, 1992)

- $\alpha\left(M_{Z}\right)=\frac{1}{127.9 \pm 0.2}$

- $\alpha_{S}\left(M_{Z}\right)=0.118 \pm 0.008$

- $\sin ^{2} \theta_{W}\left(M_{Z}\right)=0.2334 \pm 0.0008$,

and, for example, we get at one-loop

$$
\begin{aligned}
A-\frac{12}{11} B & =\frac{25}{11} \ln \frac{M_{X}}{M_{R}}+\frac{1}{11}\left[-\ln \frac{M_{X}}{m(15,2,2)_{126}}-\ln \frac{M_{X}}{m(6,1,1)_{126}}\right. \\
& \left.-4 \ln \frac{M_{X}}{m(20,1,1)_{54}}+3 \ln \frac{M_{X}}{m(1,3,3)_{54}}-\ln \frac{M_{X}}{m(6,1,1)_{10}}\right] \\
M_{R} & =M_{Z} \exp B=M_{S U(5)}
\end{aligned}
$$

(in these equations we insert all the scalars but, according to the Appelquist-Carazzone theorem (Appelquist and Carazzone, 1975), they contribute only if their mass is below $M_{X}$ ), where the coefficient of the first term on the r.h.s. of eq. (68) is given by the sum of the contributions of the gauge bosons (2), the Higgs doublets of the $10\left(2 \frac{1}{22}\right)$ 円 and the triplets of

\footnotetext{
${ }^{1}$ We take two 10 representations for the Higgses responsible of the spontaneous breaking of the electroweak theory to avoid the prediction $m_{t}=m_{b}$. The discussion on $V_{\Phi, \psi, \rho}$ of the previous section may be easily generalized to the case of two 10's.
} 
the $126 \oplus \overline{126}$ under $S U(2)_{R}(1)$ or $S U(2)_{L}\left(\frac{-9}{11}\right)$. By neglecting the term in square brackets, absent within ESH, we get at one- and two-loops respectively ${ }^{2}$ :

$$
\begin{array}{lll}
M_{R}^{(1)}=4.0 \cdot 10^{13} \cdot 1.3^{0 \pm 1} & M_{X}^{(1)}=1.0 \cdot 10^{15} \cdot 1.6^{0 \pm 1} & \tau_{p \rightarrow e^{+} \pi^{0}}^{(1)}=1.1 \cdot 10^{31} \cdot 1.6^{0 \pm 4} \\
M_{R}^{(2)}=3.4 \cdot 10^{13} & M_{X}^{(2)}=5.5 \cdot 10^{14} & \tau_{p \rightarrow e^{+} \pi^{0}}^{(2)}=8.6 \cdot 10^{29}
\end{array}
$$

in disagreement with the lower limit $\tau_{p \rightarrow e^{+} \pi^{0}} \geq 9 \cdot 10^{32}$ years.

Since $M_{R}$ is fixed by eq. (69), in order to get the highest possible value for $M_{X}$ one would like to have the highest possible value $\left(\sim M_{X}\right)$ for the $(1,3,3)$ multiplet appearing on the r.h.s. of eq. (68) and the lowest possible value $\left(\sim M_{R}\right)$ for the remaining masses. However, if the absolute minimum of the scalar potential built with the 54 is in the $G_{422} \times D$ invariant direction, eq. (8) implies $m(1,3,3)<1.35 m(20,1,1)$ (Buccella and Rosa, 1992), and consequently the contribution of the second term in the r.h.s. of eq. (68) would be $>-\frac{4}{11} \ln \frac{M_{X}}{M_{R}}-\frac{3}{11} \ln 1.35$. One would then get at one-loop

$$
M_{X}<M_{Z} e^{\frac{1}{21}(11 A+9 B+3 \ln 1.35)}=\left(2.0 \cdot 10^{15} \cdot 1.8^{0 \pm 1}\right) \mathrm{GeV}
$$

With all the multiplets at the scale $M_{R}$ but the $(1,3,3)$ one, for which we have $m(1,3,3) \leq$ $1.35 M_{R}$, one would get:

$$
\begin{array}{lll}
M_{R}^{(1)}=4.0 \cdot 10^{13} \cdot 1.3^{0 \pm 1} & M_{X}^{(1)}=2.0 \cdot 10^{15} \cdot 1.8^{0 \pm 1} & \tau_{p \rightarrow e^{+} \pi^{0}}^{(1)}=1.5 \cdot 10^{32} \cdot 1.8^{0 \pm 4} \\
M_{R}^{(2)}=3.6 \cdot 10^{13} & M_{X}^{(2)}=1.1 \cdot 10^{15} & \tau_{p \rightarrow e^{+} \pi^{0}}^{(2)}=1.2 \cdot 10^{31} .
\end{array}
$$

$M_{X}^{(2)}$ is too small (about two standard deviations) to comply with the lower limit in eq. (1).

B. $G^{\prime} \equiv S U(4)_{c} \otimes S U(2)_{L} \otimes S U(2)_{R}$

In this case, one finds

$$
\begin{aligned}
B & =\ln \frac{M_{R}}{M_{Z}}+\frac{5}{44}\left[3 \ln \frac{m(15,3,1)}{m(15,1,3)}+4 \ln \frac{m(10,3,1)}{m(10,1,3)}\right] \\
A+2 B & =\frac{\pi}{11}\left(\frac{3}{\alpha\left(M_{Z}\right)}-\frac{8}{\alpha_{S}\left(M_{Z}\right)}\right)=
\end{aligned}
$$

\footnotetext{
${ }^{2}$ The values at two-loops are the result of a numerical analysis.
} 


$$
\begin{aligned}
& =3 \ln \frac{M_{R}}{M_{Z}}+2 \ln \frac{M_{X}}{M_{R}}+\frac{1}{11}\left[\ln \frac{m(6,1,1)_{10}}{m(1,2,2)_{10}}+\frac{3}{2} \ln \frac{M_{X}^{2}}{m(15,3,1)_{210} m(15,1,3)_{210}}\right. \\
& +\ln \frac{M_{X}^{2}}{m(10,3,1)_{126} m(\overline{10}, 1,3)_{126}}-2 \ln \frac{M_{X}}{m(15,1,1)_{210}}-2 \ln \frac{M_{X}}{m(10,2,2)_{210}} \\
& \left.-\quad \ln \frac{M_{X}}{m(15,2,2)_{126}}-\ln \frac{M_{X}}{m(6,1,1)_{126}}\right] .
\end{aligned}
$$

The ESH would imply $m(1,2,2)_{10} \sim M_{Z}, m(\overline{10}, 1,3) \sim M_{R}$ and all the other scalars with mass $\sim M_{X}$. One would obtain at one- and two-loops respectively:

$$
\begin{array}{lll}
M_{R}^{(1)}=5.2 \cdot 10^{11} \cdot 2.1^{0 \pm 1} & M_{X}^{(1)}=7.1 \cdot 10^{15} \cdot 1.9^{0 \pm 1} & \tau_{p \rightarrow e^{+} \pi^{0}}^{(1)}=2.5 \cdot 10^{34} \cdot 1.9^{0 \pm 4} \\
M_{R}^{(2)}=1.4 \cdot 10^{11} & M_{X}^{(2)}=5.3 \cdot 10^{15} & \tau_{p \rightarrow e^{+} \pi^{0}}^{(2)}=7.6 \cdot 10^{33}
\end{array}
$$

The value found for $M_{R}$ is lower than $M_{S U(5)}$ and it is phenomenologically intriguing (Mohapatra and Parida, 1993; Babu and Shafi, 1993). To establish how much the value found depends on ESH, we look for the highest value for $M_{R}$ consistent with eq. (1).

From eqs. (71) and (42), by taking $m(10,3,1) \geq M_{R}$, one gets

$$
M_{R}<M_{Z} e^{B} e^{\frac{15}{88} \ln \frac{2+\sqrt{3}}{2-\sqrt{3}}}=\left(6.2 \cdot 10^{13} \cdot 1.4^{0 \pm 1}\right) \mathrm{GeV} .
$$

$M_{R}$ takes the value $6.2 \cdot 10^{13} \mathrm{GeV}$ if

$$
\begin{aligned}
m(10,3,1)_{210} & =m(\overline{10}, 1,3)_{210} \\
m^{2}(15,3,1)_{210} & =\frac{2-\sqrt{3}}{2+\sqrt{3}} m^{2}(15,1,3)_{210}
\end{aligned}
$$

which corresponds to $\beta=-\frac{3}{8}(\alpha+2 \gamma)$, just on the boundary of the allowed values for $\beta$ that are compatible with the absolute minimum being in the $G_{422}$-invariant direction. For that value of $\beta, \alpha$ should be $\leq 2 \gamma$ and one has the inequality

$$
\frac{m^{2}(15,3,1)_{210} m^{2}(15,1,3)_{210}}{m^{4}(15,1,1)_{210}}=\frac{9(\alpha+2 \gamma)^{2}}{16 \gamma^{2}} \leq 9 .
$$

From eqs. (72), (74), (75), and (76) one gets:

$$
M_{R} \leq 3^{\frac{1}{14}} M_{Z}\left(\frac{M_{Z}}{M_{X}}\right)^{\frac{10}{7}} e^{\frac{\pi}{14}\left(\frac{3}{\alpha^{\left(M_{Z}\right)}-\frac{8}{\alpha_{S}\left(M_{Z}\right)}}\right)} \leq 3 \cdot 10^{13} \mathrm{GeV},
$$


the last inequality coming from eq. (11).

The highest value for $M_{R}$ consistent with eq. (1) is found by taking $(1,2,2)_{10}$ at the scale $M_{Z}$, the multiplets $(10,2,2)_{210},(6,1,1)_{10}$ and all the states of the 126 at the scale $M_{R}$ and all the other states of the 210 at the scale $M_{X}$; in such conditions the numerical analysis gives:

$$
\begin{array}{lll}
M_{R}^{(1)}=1.6 \cdot 10^{13} \cdot 2.5^{0 \pm 1} & M_{X}^{(1)}=4.7 \cdot 10^{15} \cdot 1.8^{0 \pm 1} & \tau_{p \rightarrow e^{+} \pi^{0}}^{(1)}=4.8 \cdot 10^{33} \cdot 1.8^{0 \pm 4} \\
M_{R}^{(2)}=1.1 \cdot 10^{13} & M_{X}^{(2)}=3.1 \cdot 10^{15} & \tau_{p \rightarrow e^{+} \pi^{0}}^{(2)}=9.2 \cdot 10^{32} .
\end{array}
$$

C. $G^{\prime} \equiv S U(3)_{c} \otimes S U(2)_{L} \otimes S U(2)_{R} \otimes U(1)_{B-L} \times D$

In this case, by keeping into account the $S U(2)_{L} \leftrightarrow S U(2)_{R}$ symmetry above $M_{R}$, we get

$$
\begin{aligned}
2 B-A & =\ln \frac{M_{R}}{M_{Z}}+\frac{1}{11}\left[-\ln \frac{M_{X}}{m(1,2,2,0)_{10}}+\ln \frac{M_{X}}{m(1,3,1,-2)_{126}}+2 \ln \frac{m(8,3,1,0)_{210}}{m(6,2,2,2 / 3)_{210}}\right. \\
& +\ln \frac{M_{X}}{m(1,2,2,2)_{210}}+2 \ln \frac{m(3,3,1,-2 / 3)_{126}}{m(3,2,2,4 / 3)_{126}}+\ln \frac{m(3,3,1,-2 / 3)_{126}}{m(3,1,1,-2 / 3)_{126}} \\
& \left.+\ln \frac{m(3,2,2,-2 / 3)_{210}}{m(8,1,1,0)_{210}}+\ln \frac{m(1,2,2,0)_{126}}{m(3,1,1,-2 / 3)_{126}}\right] \\
B & =\frac{1}{2} \ln \frac{M_{X} M_{R}}{M_{Z}^{2}}+\frac{1}{11}\left[\frac{3}{2} \ln \frac{M_{X}}{m(1,3,1,-2)_{126}}+\ln \frac{m(6,2,2,2 / 3)_{210}}{m(1,2,2,2)_{210}}\right. \\
& +\frac{3}{2} \ln \frac{m(8,3,1,0)_{210}}{m(3,3,1,4 / 3)_{210}}-\frac{1}{4} \ln \frac{M_{X}}{m(8,1,1,0)_{210}}+2 \ln \frac{m(8,2,2,0)_{126}}{m(3,2,2,4 / 3)_{126}} \\
& \left.-\frac{3}{2} \ln \frac{M_{X}}{m(6,3,1,2 / 3)_{126}}\right] .
\end{aligned}
$$

The ESH would imply $m(1,2,2,0)_{10} \sim M_{Z}, m(1,3,1,-2)_{126} \sim M_{R}$ and all the other multiplets at the scale $M_{X}$. One should get at one- and two-loops

$$
\begin{array}{lll}
M_{R}^{(1)}=1.2 \cdot 10^{10} \cdot 1.8^{0 \pm 1} & M_{X}^{(1)}=4.1 \cdot 10^{15} \cdot 2.8^{0 \pm 1} & \tau_{p \rightarrow e^{+} \pi^{0}}^{(1)}=2.8 \cdot 10^{33} \cdot 2.8^{0 \pm 4} \\
M_{R}^{(2)}=3.2 \cdot 10^{10} & M_{X}^{(2)}=1.6 \cdot 10^{15} & \tau_{p \rightarrow e^{+} \pi^{0}}^{(2)}=7.0 \cdot 10^{31}
\end{array}
$$

a too small value for $\tau_{p \rightarrow e^{+} \pi^{0}}^{(2)}$.

In considering the contribution of the other scalars we take into account the constraints on the mass spectrum, which follow from the requirement that the absolute minimum falls in 
the desired direction. The parameters defined in (27) should obey eq. (53), which implies the following inequalities for the masses (the case discussed here is obtained with $z_{1}=1 z_{3}=0$ ):

$$
\frac{m(8,3,1,0)_{210}}{m(3,3,1,4 / 3)_{210}}>\sqrt{\frac{37}{14}}, \quad \frac{m(6,2,2,2 / 3)_{210}}{m(1,2,2,2)_{210}}>\frac{1}{\sqrt{7}}, \quad 1<\frac{m(8,3,1,0)_{210}}{m(6,2,2,2 / 3)_{210}}<\frac{2}{\sqrt{3}} .
$$

From the positivity of the $f_{i}$ 's defined in eq. 21) and from the expressions for the masses in the Appendix one gets also

$$
1 \leq \frac{m(3,3,1,-2 / 3)_{126}}{m(3,2,2,4 / 3)_{126}} \leq 2
$$

From eqs. (78), (79), (80), and (81) one finds the inequalities

$$
\begin{aligned}
& M_{R} \leq\left(\frac{2 \cdot 7^{\frac{5}{3}}}{37}\right)^{\frac{3}{31}} \frac{M_{S U(5)}^{\frac{44}{31}}}{M_{X}^{\frac{13}{31}}} \\
& M_{R} \leq M_{Z}^{\frac{5}{6}} M_{X}^{\frac{1}{6}} e^{\frac{\pi}{\alpha\left(M_{Z}\right)}\left(\frac{1}{4}-\sin ^{2} \theta_{W}\left(M_{Z}\right)+\frac{1}{3} \frac{\alpha}{\alpha_{S}}\left(M_{Z}\right)\right) .}
\end{aligned}
$$

The highest value for $M_{R}$ is found by taking $m(8,1,1,0)_{210}, m(6,3,1,2 / 3)_{126}$ and $m(8,2,2,0)_{126}$ at the scale $M_{R}$ and $m(3,2,2,4 / 3)_{126}$ at the scale $M_{X}$ for eq. (82), and $m(3,2,2,-2 / 3)_{210}, m(3,3,1,-2 / 3)_{126}$, and $m(3,2,2,4 / 3)_{126}$ at the scale $M_{R}, m(1,2,2,2)_{210}$, $m(8,1,1,0)_{210}, m(3,1,1,-2 / 3)_{126}$, and $m(1,2,2,0)_{126}$ at the scale $M_{X}$, and $m(8,3,1,0)_{210}=$ $m(6,2,2,2 / 3)_{210}$ for eq. (83). The two requirements may not be satisfied at the same time, since they imply a different scale for $m(8,1,1,0)_{210}$ and $m(3,2,2,4 / 3)_{126}$. Except for the factor $\left(\frac{2 \cdot 7^{\frac{5}{3}}}{37}\right)^{\frac{3}{31}} \sim 1.03$, eq. (82) is the same found in Buccella and Rosa (1992) with more restrictive conditions.

By eliminating $M_{X}$ in eqs. (82) and (83) one finds the inequality

$$
M_{R}<3.1 \cdot 10^{11} \cdot 3.1^{0 \pm 1} \mathrm{GeV} .
$$

Certainly, it would be possible to get a lower bound for $M_{R}$ since the one just written has been obtained by multiplying inequalities which cannot be both equalities. So we are not surprised when, by looking for the highest value for $M_{R}$ consistent with eq. (11) and with the 
constraints on the spectrum following from eq. (53), the numerical analysis gives:

$$
\begin{array}{lll}
M_{R}^{(1)}=2.4 \cdot 10^{10} \cdot 3.1^{0 \pm 1} & M_{X}^{(1)}=8.1 \cdot 10^{15} \cdot 1.9^{0 \pm 1} & \tau_{p \rightarrow e^{+} \pi^{0}}^{(1)}=4.1 \cdot 10^{34} \cdot 1.9^{0 \pm 4} \\
M_{R}^{(2)}=2.9 \cdot 10^{10} & M_{X}^{(2)}=3.1 \cdot 10^{15} & \tau_{p \rightarrow e^{+} \pi^{0}}^{(2)}=9.3 \cdot 10^{32}
\end{array}
$$

with $M_{R}^{(1)}$ lower than the r.h.s. of eq. (84).

D. $G^{\prime} \equiv S U(3)_{c} \otimes S U(2)_{L} \otimes S U(2)_{R} \otimes U(1)_{B-L}$

In this case, the absence of D symmetry brings to more complicate expressions for A and B, namely

$$
\begin{aligned}
A & =\ln \frac{M_{X}}{M_{Z}}+\frac{1}{11}\left[\ln \frac{M_{X}}{m(1,2,2,0)_{10}}+\ln \frac{m(8,3,1,0)_{210}}{m(3,3,1,4 / 3)_{210}}+\ln \frac{m(6,2,2,2 / 3)_{210}}{m(1,2,2,2)_{210}}\right. \\
& +2 \ln \frac{m(6,2,2,2 / 3)_{210}}{m(3,3,1,4 / 3)_{210}}+\ln \frac{m(6,2,2,2 / 3)_{210}}{m(3,2,2,-2 / 3)_{210}}-\frac{3}{2} \ln \frac{M_{X}}{m(8,1,1,0)_{210}} \\
& +2 \ln \frac{M_{X}}{m(1,3,1,-2)_{126}}-\ln \frac{M_{X}}{m(3,1,1,-2 / 3)_{126}}+2 \ln \frac{m(8,2,2,0)_{126}}{m(3,2,2,4 / 3)_{126}} \\
& \left.+3 \ln \frac{m(6,3,1,2 / 3)_{126}}{m(3,3,1,-2 / 3)_{126}}+\ln \frac{m(3,1,1,-2 / 3)_{126}}{m(1,2,2,0)_{126}}-2 \ln \frac{M_{X}}{m(8,2,2,0)_{126}}\right] \\
& +\frac{1}{11}\left[\ln \frac{M_{X}}{m(1,3,1,0)_{210}}+\frac{9}{2} \ln \frac{m(8,1,3,0)_{210}}{m(8,3,1,0)_{210}}+\frac{3}{2} \ln \frac{m(3,1,3,4 / 3)_{210}}{m(3,3,1,4 / 3)_{210}}\right. \\
& +\frac{15}{2} \ln \frac{m(\overline{6}, 1,3,-2 / 3)_{126}}{m(6,3,1,2 / 3)_{126}}+\frac{3}{2} \ln \frac{m(3,1,3,2 / 3)_{126}}{m(3,3,1,-2 / 3)_{126}}+\ln \frac{m(3,2,2,4 / 3)_{126}}{m(\overline{3}, 2,2,-4 / 3)_{126}} \\
& \left.+\frac{1}{2} \ln \frac{m(\overline{3}, 1,1,2 / 3)_{126}}{m(3,1,1,-2 / 3)_{126}}+\ln \frac{m(3,1,1,-2 / 3)_{10}}{m(3,1,1,-2 / 3)_{126}}\right] \\
& =\frac{1}{2} \ln \frac{M_{X} M_{R}}{M_{Z}^{2}}+\frac{1}{11}\left[\ln \frac{m(6,2,2,2 / 3)_{210}}{m(1,2,2,2)_{210}}+\frac{3}{2} \ln \frac{m(8,3,1,0)_{210}}{m(3,3,1,4 / 3)_{210}}\right. \\
& -\frac{1}{4} \ln \frac{M_{X}}{m(8,1,1,0)_{210}}+\frac{3}{2} \ln \frac{M_{X}}{m(1,3,1,-2)_{126}}+2 \ln \frac{m(8,2,2,0)_{126}}{m(3,2,2,4 / 3)_{126}} \\
& \left.-\frac{3}{2} \ln \frac{M_{X}}{m(6,3,1,2 / 3)_{126}}\right]+\frac{1}{11}\left[\frac{5}{4} \ln \frac{M_{X}}{m(1,1,3,2)_{126}}+\frac{5}{4} \ln \frac{m(8,3,1,0)_{210}}{m(8,1,3,0)_{210}}\right. \\
& +\frac{9}{4} \ln \frac{m(3,3,1,4 / 3)_{210}}{m(3,1,3,4 / 3)_{210}}+\frac{1}{4} \ln \frac{m(1,3,1,0)_{210}}{m(1,1,3,0)_{210}}-\frac{5}{4} \ln \frac{M_{X}}{m(1,3,1,-2)_{126}} \\
& \left.+\frac{9}{4} \ln \frac{m(6,3,1,2 / 3)_{126}}{m(\overline{6}, 1,3,-2 / 3)_{126}}+\frac{3}{2} \ln \frac{m(3,3,1,-2 / 3)_{126}}{m(\overline{3}, 1,3,2 / 3)_{126}}+\ln \frac{m(3,2,2,4 / 3)_{126}}{m(\overline{3}, 2,2,-4 / 3)_{126}}\right] .
\end{aligned}
$$

At one- and two-loops, one should get, with the ESH:

$$
\begin{array}{lll}
M_{R}^{(1)}=8.3 \cdot 10^{8} \cdot 3.3^{0 \pm 1} & M_{X}^{(1)}=3.5 \cdot 10^{16} \cdot 2.1^{0 \pm 1} & \tau_{p \rightarrow e^{+} \pi^{0}}^{(1)}=1.4 \cdot 10^{37} \cdot 2.1^{0 \pm 4} \\
M_{R}^{(2)}=2.7 \cdot 10^{9} & M_{X}^{(2)}=1.1 \cdot 10^{16} & \tau_{p \rightarrow e^{+} \pi^{0}}^{(2)}=1.5 \cdot 10^{35} .
\end{array}
$$


In a previous paper (Buccella and Rosa, 1992) one considered a particular direction,

$$
\Phi_{A B R S} \equiv \Phi_{0}\left(\sqrt{\frac{3}{5}}, 0, \sqrt{\frac{2}{5}}\right)=\sqrt{\frac{3}{5}} \Phi_{L}+\sqrt{\frac{2}{5}} \Phi_{T},
$$

for the breaking at the highest scale and, for the model introduced in Abud, Buccella, Rosa, and Sciarrino (1989), one has been able to deduce, from the inequalities found for the masses and eq. (86), the inequality

$$
M_{R} \leq \frac{M_{S U(5)}^{2}}{M_{X}} \leq 5 \cdot 10^{11} \mathrm{GeV},
$$

the last inequality being deduced from the lower limit on $M_{X}$ in eq. (四).

One expects to find the absolute minimum of $V_{\Phi}$ around the direction $\Phi_{A B R S}$ if the coefficient of the invariant $\left\|(\Phi \Phi)_{54}\right\|$, vanishing at $\Phi_{A B R S}$, is positive and larger than the others. Indeed, it can be shown that for $\beta \neq 0$ one can get values of the parameters consistent with $\tilde{z}_{1}=\frac{\beta+\sqrt{\beta^{2}-\alpha \gamma}}{\alpha}=\sqrt{\frac{3}{5}}$ and with the necessary conditions described in table III.

Here, we are looking for the highest value for $M_{R}$ consistent with the necessary conditions obtained in the thesis of one of us (Pisanti, 1992) to get the minimum in the desired direction.

Due to the complexity of the conditions, we have not been able to deduce analytically, as in the previous cases, interesting inequalities for the one-loop equations. It has been therefore necessary to proceed numerically (Rosa, 1993) to the search of the highest value for $M_{R}$. The following predictions for $M_{R}, M_{X}$, and $\tau_{p \rightarrow e^{+} \pi^{0}}$, which were obtained with the scalars of the $126 \oplus \overline{126}$ at the scale $M_{R}$ and with the scalars of the 210 at the scale $M_{X}$, are the result of this numerical analysis:

$$
\begin{array}{lll}
M_{R}^{(1)}=4.8 \cdot 10^{10} \cdot 2.5^{0 \pm 1} & M_{X}^{(1)}=2.8 \cdot 10^{16} \cdot 2.0^{0 \pm 1} & \tau_{p \rightarrow e^{+} \pi^{0}}^{(1)}=6.1 \cdot 10^{36} \cdot 2.0^{0 \pm 4} \\
M_{R}^{(2)}=1.2 \cdot 10^{11} & M_{X}^{(2)}=1.9 \cdot 10^{16} & \tau_{p \rightarrow e^{+} \pi^{0}}^{(2)}=1.2 \cdot 10^{36}
\end{array}
$$

\section{Conclusions}

We have studied the predictions for the values of the scale of spontaneous breaking of the intermediate symmetry $G^{\prime} \supset S U(2)_{R}$ for a class of $S O(10)$ models. In table IV we report 
TABLE IV

\begin{tabular}{|c|c|c|}
\hline$\frac{G^{\prime}}{S U(2)_{R} \otimes S U(2)_{L}}$ & $\frac{M_{R}^{(2)}}{10^{11} G e V}$ & $\frac{M_{X}^{(2)}}{3.2 \cdot 10^{15} \mathrm{GeV}}$ \\
\hline$S U(4)_{P S} \times D$ & $360 \cdot(1.3)^{0 \pm 1}$ & $0.34 \cdot(1.8)^{0 \pm 1}$ \\
\hline$S U(4)_{P S}$ & $110 \cdot(2.5)^{0 \pm 1}$ & $0.97 \cdot(1.8)^{0 \pm 1}$ \\
\hline$S U(3)_{C} \otimes U(1)_{B-L} \times D$ & $0.29 \cdot(3.1)^{0 \pm 1}$ & $0.97 \cdot(1.9)^{0 \pm 1}$ \\
\hline$S U(3)_{C} \otimes U(1)_{B-L}$ & $1.2 \cdot(2.5)^{0 \pm 1}$ & $5.9 \cdot(2.0)^{0 \pm 1}$ \\
\hline
\end{tabular}

the values of the upper limits found for $M_{R}$ by demanding that, from proton stability, $M_{X} \geq$ $3.2 \cdot 10^{15} \mathrm{GeV}$.

For the model with $G^{\prime} \supset S U(4)_{P S} \times D$, which is the one giving rise to the largest value for $M_{R}$, the central prediction for $M_{X}$ is two standard deviations away from the experimental lower limit.

The upper limit for $M_{R}$ for the model with $\frac{G^{\prime}}{S U(2)_{R} \otimes S U(2)_{L}}=S U(4)_{P S}$ is almost two orders of magnitude larger than the value found with the ESH.

For the last two models we find an upper limit for $M_{R}\left(=1.2 \cdot 10^{11} \cdot(2.5)^{0 \pm 1} \mathrm{GeV}\right)$ which gives rise, within the see-saw mechanism, to the following lower limits for $m_{\nu_{\tau}}$ and $m_{\nu_{\mu}}$ :

$$
\begin{aligned}
& m_{\nu_{\tau}} \geq 11 \frac{g_{2 R}\left(M_{R}\right)}{f_{3}\left(M_{R}\right)}\left(\frac{m_{t}}{100 G e V}\right)^{2} \mathrm{eV} \\
& m_{\nu_{\mu}} \geq 2.4 \cdot 10^{-3} \frac{g_{2 R}\left(M_{R}\right)}{f_{2}\left(M_{R}\right)} \mathrm{eV} .
\end{aligned}
$$

(these formulae are obtained using the following values: $m_{\tau}=1784.1 \mathrm{MeV}, m_{c}=1500 \mathrm{MeV}$, $m_{b}=5000 \mathrm{MeV}$ (Review of Particle Properties, 1992); $g_{2 R}$ and $f_{i}$ are the $S U(2)_{R}$ gauge coupling constant and the Yukawa coupling of the $126 \oplus \overline{126}$ to the i-th family respectively.)

These values imply a substantial contribution of $\nu_{\tau}$ to the dark matter in the universe and a value for $\nu_{\mu}$ which might be relevant for the solution of the solar-neutrino problem in 
terms of the MSW model. With respect to a previous analysis by two of us (Buccella and Rosa, 1992) the results for the model with $G^{\prime} \supset S U(4)_{P S} \times D$ are modified mainly by the recent slight increase in the experimental value of $\alpha_{s}\left(M_{Z}\right)$.

For the models with $G^{\prime} \supset S U(4)_{P S}$ or $S U(3)_{C} \otimes U(1)_{B-L} \times D$ the region allowed for the parameters is reduced by the necessary requirement that the absolute minimum in the 3-dimensional $S U(3)_{c} \otimes S U(2)_{L} \otimes U(1)_{T_{3 R}} \otimes U(1)_{B-L}$-stratum is $V\left(\Phi_{T}\right)$ or $V\left(\Phi_{L}\right)$ respectively.

For the model with $G^{\prime} \equiv G_{3221}$ the present analysis concerns all values of the parameters complying with the conditions which are necessary for the desired symmetry breaking pattern, while in Buccella and Rosa (1992) only the particular choice of Abud, Buccella, Rosa, and Sciarrino (1989) was considered.

The conclusion of our rather general analysis of the $S O(10)$ models is that by requiring agreement with the present lower limit for $\tau_{p \rightarrow e^{+} \pi^{0}}$ one finds neutrino masses for $\nu_{\tau}$ and $\nu_{\mu}$ of the order of magnitude relevant for cosmology and solar-neutrino astrophysics.

The model with $G^{\prime} \supset S U(4)_{P S} \times D$, for which smaller $\nu_{\tau}$ masses are expected, is almost excluded by experimental information on proton decay.

The model with $G^{\prime} \supset S U(4)_{P S}$, which however within the ESH predicts a value of $m_{\nu_{\mu}}=$ $2.0 \cdot 10^{-3} \frac{g_{2 R}\left(M_{R}\right)}{f_{2}\left(M_{R}\right)} \mathrm{eV}$, may predict lower values $\left(\sim 10^{-5} \frac{g_{2 R}\left(M_{R}\right)}{f_{2}\left(M_{R}\right)} \mathrm{eV}\right)$ only if ad-hoc assumptions for the masses of the $S U(2)_{L(R)}$ triplets of the 126 are made, while the contribution to RGE of the triplets of the 210 is controlled by the necessary condition in eq. (42).

A better knowledge of the gauge coupling constants at $M_{Z}$, especially of $\alpha_{S}$, as well as the increase of the lower limit on $\tau_{p \rightarrow e^{+} \pi^{0}}$ would improve the predictive power of the analysis here described.

\section{Appendix}

$$
\frac{m^{2}(8,3,1,0)}{r^{2}}=\alpha\left(\frac{3}{4}-\frac{3}{4} z_{1}^{2}-\frac{1}{2} z_{1}^{4}+\frac{\sqrt{3}}{2} z_{1} z_{3}\right)+\beta\left(z_{1}+z_{1}^{3}+\sqrt{3} z_{3}\right)
$$




$$
\begin{aligned}
& +\gamma\left(\frac{3}{2}-\frac{5}{2} z_{1}^{2}+\frac{7}{2 \sqrt{3}} z_{1} z_{3}\right)+\delta\left(-\frac{40}{9} z_{1}^{2}+\frac{40}{3 \sqrt{3}} z_{1} z_{3}\right) \\
& \frac{m^{2}(8,1,3,0)}{r^{2}}=\alpha\left(\frac{3}{4}-\frac{3}{4} z_{1}^{2}-\frac{1}{2} z_{1}^{4}-\frac{\sqrt{3}}{2} z_{1} z_{3}\right)+\beta\left(z_{1}+z_{1}^{3}-\sqrt{3} z_{3}\right) \\
& +\gamma\left(\frac{3}{2}-\frac{5}{2} z_{1}^{2}-\frac{7}{2 \sqrt{3}} z_{1} z_{3}\right)-\delta\left(\frac{40}{9} z_{1}^{2}+\frac{40}{3 \sqrt{3}} z_{1} z_{3}\right) \\
& \frac{m^{2}(3,3,1,4 / 3)}{r^{2}}=\alpha\left(\frac{3}{4}-\frac{1}{4} z_{1}^{2}-\frac{1}{2} z_{1}^{4}-\frac{\sqrt{3}}{2} z_{1} z_{3}\right)+\beta\left(-z_{1}+z_{1}^{3}+\sqrt{3} z_{3}\right) \\
& +\gamma\left(\frac{3}{2}-\frac{3}{2} z_{1}^{2}-\frac{7}{2 \sqrt{3}} z_{1} z_{3}\right)-\delta\left(\frac{20}{9} z_{1}^{2}+\frac{40}{3 \sqrt{3}} z_{1} z_{3}\right) \\
& \frac{m^{2}(3,1,3,4 / 3)}{r^{2}}=\alpha\left(\frac{3}{4}-\frac{1}{4} z_{1}^{2}-\frac{1}{2} z_{1}^{4}+\frac{\sqrt{3}}{2} z_{1} z_{3}\right)+\beta\left(-z_{1}+z_{1}^{3}-\sqrt{3} z_{3}\right) \\
& +\gamma\left(\frac{3}{2}-\frac{3}{2} z_{1}^{2}+\frac{7}{2 \sqrt{3}} z_{1} z_{3}\right)-\delta\left(\frac{20}{9} z_{1}^{2}-\frac{40}{3 \sqrt{3}} z_{1} z_{3}\right) \\
& \frac{m^{2}(1,3,1,0)}{r^{2}}=\alpha\left(\frac{3}{4}+\frac{3}{4} z_{1}^{2}-\frac{1}{2} z_{1}^{4}-\sqrt{3} z_{1} z_{3}\right)+\beta\left(-2 z_{1}+z_{1}^{3}+\sqrt{3} z_{3}\right) \\
& +\gamma\left(\frac{3}{2}+z_{1}^{2}-\frac{7}{\sqrt{3}} z_{1} z_{3}\right)+\delta\left(\frac{80}{9} z_{1}^{2}-\frac{80}{3 \sqrt{3}} z_{1} z_{3}\right) \\
& \frac{m^{2}(1,1,3,0)}{r^{2}}=\alpha\left(\frac{3}{4}+\frac{3}{4} z_{1}^{2}-\frac{1}{2} z_{1}^{4}+\sqrt{3} z_{1} z_{3}\right)+\beta\left(-2 z_{1}+z_{1}^{3}-\sqrt{3} z_{3}\right) \\
& +\gamma\left(\frac{3}{2}+z_{1}^{2}+\frac{7}{\sqrt{3}} z_{1} z_{3}\right)+\delta\left(\frac{80}{9} z_{1}^{2}+\frac{80}{3 \sqrt{3}} z_{1} z_{3}\right) \\
& \frac{m^{2}(8,1,1,0)}{r^{2}}=-\frac{1}{2} \alpha z_{1}^{4}+\beta\left(z_{1}+z_{1}^{3}\right)+\frac{1}{2} \gamma\left(1-3 z_{1}^{2}\right) \\
& \frac{m^{2}(3,1,1,4 / 3)}{r^{2}}=\frac{1}{2} \alpha\left(z_{1}^{2}-z_{1}^{4}\right)+\beta\left(-z_{1}+z_{1}^{3}\right)+\frac{1}{2} \gamma\left(1-z_{1}^{2}\right) \\
& \frac{m^{2}(6,2,2,2 / 3)}{r^{2}}=-\frac{1}{2} \alpha z_{1}^{4}+\beta\left(z_{1}+z_{1}^{3}\right)-\gamma z_{1}^{2}+\delta\left(-5+\frac{5}{3} z_{1}^{2}\right)
\end{aligned}
$$




$$
\begin{gathered}
\frac{m^{2}(1,2,2,2)}{r^{2}}=\alpha\left(3 z_{1}^{2}-\frac{1}{2} z_{1}^{4}\right)+\beta\left(-3 z_{1}+z_{1}^{3}\right)+\gamma 5 z_{1}^{2}+\delta\left(-5+15 z_{1}^{2}\right) \\
\frac{m^{2}(3,2,2,-2 / 3)_{d i a g}}{r^{2}}= \begin{cases}\frac{(\gamma+10 \delta)\left(-3+z_{1}^{2}\right)}{6} & S U(3)_{c} \otimes S U(2)_{L} \otimes S U(2)_{R} \otimes U(1)_{B-L} \\
-5 \delta & S U(4)_{P S} \otimes S U(2)_{L} \otimes S U(2)_{R} \\
\frac{\left(-\alpha+2 \beta-\frac{5}{3} \gamma-\frac{20}{3} \delta\right)}{2} & S U(3)_{c} \otimes S U(2)_{L} \otimes S U(2)_{R} \otimes U(1)_{B-L} \times D\end{cases}
\end{gathered}
$$$$
126 \oplus \overline{126}
$$

$$
\begin{aligned}
& \frac{m^{2}(6,3,1,2 / 3)}{s^{2}}=f_{4} \frac{1}{75}\left(2 z_{1}+\sqrt{3} z_{3}\right)^{2} \\
& \frac{m^{2}(3,3,1,-2 / 3)}{s^{2}}=f_{2} \frac{4}{315} z_{1}^{2}+f_{3} \frac{8}{315} z_{1}^{2}+f_{4} \frac{1}{75}\left(z_{1}+\sqrt{3} z_{3}\right)^{2} \\
& \frac{m^{2}(1,3,1,-2)}{s^{2}}=f_{4} \frac{1}{25} z_{3}^{2} \\
& \frac{m^{2}(8,2,2,0)}{s^{2}}=f_{2} \frac{1}{105} z_{3}^{2}+f_{3} \frac{2}{105}\left(z_{1}+z_{3}\right)^{2}+f_{4} \frac{1}{300}\left(4 z_{1}+\sqrt{3} z_{3}\right)^{2} \\
& \frac{m^{2}(3,2,2,4 / 3)}{s^{2}}=f_{2} \frac{1}{105}\left(z_{3}+\sqrt{\frac{4}{3}} z_{1}\right)^{2}+f_{3} \frac{2}{315}\left(z_{1}-\sqrt{3} z_{3}\right)^{2}+f_{4} \frac{1}{300}\left(2 z_{1}+\sqrt{3} z_{3}\right)^{2} \\
& \frac{m^{2}(\overline{3}, 2,2,-4 / 3)}{s^{2}}=f_{2} \frac{1}{105}\left(z_{3}-\sqrt{\frac{4}{3}} z_{1}\right)^{2}+f_{3} \frac{2}{315}\left(z_{1}+\sqrt{3} z_{3}\right)^{2}+f_{4} \frac{1}{300}\left(2 z_{1}+\sqrt{3} z_{3}\right)^{2} \\
& \frac{m^{2}(1,2,2,0)}{s^{2}}=f_{1} \frac{1}{126} z_{1}^{2}+f_{2} \frac{1}{105} z_{3}^{2}+f_{3} \frac{2}{105}\left(z_{3}+\sqrt{\frac{4}{3}} z_{1}\right)^{2}+f_{4} \frac{1}{300}\left(z_{1}+\sqrt{3} z_{3}\right)^{2} \\
& \frac{m^{2}(\overline{6}, 1,3,-2 / 3)}{s^{2}}=f_{4} \frac{4}{75} z_{1}^{2}
\end{aligned}
$$




$$
\begin{aligned}
\frac{m^{2}(\overline{3}, 1,3,2 / 3)}{s^{2}} & =f_{2} \frac{4}{315} z_{1}^{2}+f_{3} \frac{8}{315} z_{1}^{2}+f_{4} \frac{1}{75} z_{1}^{2} \\
\frac{m^{2}(3,1,1,-2 / 3)}{s^{2}} & =f_{1} \frac{1}{378}\left(z_{1}-\sqrt{3} z_{3}\right)^{2}+f_{2} \frac{2}{315} z_{1}^{2}+f_{3} \frac{4}{63}\left(z_{3}+\frac{4}{\sqrt{15}} z_{1}\right)^{2}+f_{4} \frac{1}{100}\left(z_{3}+\sqrt{3} z_{1}\right)^{2} \\
\frac{m^{2}(\overline{3}, 1,1,2 / 3)}{s^{2}} & =f_{1} \frac{1}{378}\left(z_{1}+\sqrt{3} z_{3}\right)^{2}+f_{2} \frac{2}{315} z_{1}^{2}+f_{3} \frac{4}{63}\left(z_{3}-\frac{4}{\sqrt{15}} z_{1}\right)^{2}+f_{4} \frac{1}{100}\left(z_{3}+\sqrt{3} z_{1}\right)^{2}
\end{aligned}
$$

\section{References}

Abud, M., F. Buccella, A. Della Selva, A. Sciarrino, R. Fiore, and G. Immirzi, 1986, Nucl. Phys. B 263, 336.

Abud, M., F. Buccella, L. Rosa, A. Sciarrino, 1989, Zeitschrift für Phys. C 44, 589.

Amaldi, U., W. de Boer, and H. Furstenau, 1991, Phys. Lett. B 260, 447.

Amelino-Camelia, G., 1989, "Spontaneous Symmetry Breaking in SO(10) GUT Models with $\tau_{p} \sim 10^{33}$ years" First-Degree Thesis (unpublished).

Amelino-Camelia, G., F. Buccella, and L. Rosa, 1990, Atti del "Second International Workshop on Neutrino Telescopes" ed. Milla Baldo Ceolin.

Anselmo, F., L. Cifarelli, and A. Zichichi, 1992, Il Nuovo Cimento A 105 n.9, 1357.

Appelquist,T., and J. Carazzone, 1975, Phys. Rev. D 11 n.10, 2856.

Babu, K. S., and Q. Shafi, 1993, Nucl. Phys. B (Proc. Suppl.) 31, 242.

Barbieri, R., G. Morchio, D.V. Nanopoulos, and F. Strocchi, 1980, Phys. Lett. B 90, 91.

Baseq, J., S. Meljanac, and L. O'Raifeartaigh, 1989, Phys. Rev. D 39, 3110.

Buccella, F., 1988, in "Symmetry in Science III", eds. B. Gruber. and F. Iachello.

Buccella, F., L. Cocco, A. Sciarrino, and T. Tuzi, 1986, Nucl. Phys. B 274, 559.

Buccella, F., L. Cocco, and C. Wetterich, 1984, Nucl. Phys. B 243, 273.

Buccella, F., G. Miele, L. Rosa, P. Santorelli, and T. Tuzi, 1989, Phys. Lett. B 233, 178.

Buccella, F., and L. Rosa, 1987, Zeitschrift für Phys. C 36, 425.

Buccella, F., and L. Rosa, 1992, Nucl. Phys. B (Proc. Suppl.) 28 A, 168. 
Buccella, F., and H. Ruegg, 1982, Il Nuovo Cimento A 67 n.1, 61.

Burzlaff, J., and L. O'Raifeartaigh, 1990, Proceedings of Capri Symposia, 1983-1987, ed. F. Buccella.

Chang, D., J. M. Gipson, R. E. Marshak, R. N. Mohapatra, and M. K. Parida, 1985, Phys. Rev. D 31, 1718.

Chang, D., R. N. Mohapatra, and M. K. Parida, 1984, Phys. Rev. Lett. 52, 1072.

Deshpande, N. G., E. Keith, and Palash B. Pal, 1992, Phys. Rev. D 46 n.5, 2261.

Dixit, V. V., and M. Sher, 1989, Phys. Rev. D 40 n.11, 3765.

Fritzsch, H., and P. Minkowski, 1975, Ann. of Phys. 93, 183.

Gell-Mann, M., P. Ramond, and R. Slansky, 1980, "Supergravity" (North Holland, Amsterdam).

Georgi, H., 1975, "Particles and Fields" C. E. Carlson (AJP).

Jones, D. R. T., 1982, Phys. Rev. D 25 n.2, 581.

Kaymackcalan, O., L. Michel, K.C. Wali, W.D. Mc GLinn, and L. O'Raifeartaigh, 1986, Nucl. Phys. B 267 n.1, 203.

Kuzmin, V., and N. Shaposhnikov, 1980, Phys. Lett. B 92, 115.

Mohapatra, R. N., and M.K. Parida, 1993, Phys. Rev. D 47, 264.

Pati, J. C., and A. Salam, 1974, Phys. Rev. D 10, 275.

Pisanti, O., 1992, "An Analysis of SO(10) Models with Intermediate Symmetry $S U(3)_{c} \otimes$ $S U(2)_{L} \otimes S U(2)_{R} \otimes U(1)_{B-L} "$ First-Degree Thesis (unpublished).

Review of Particle Properties, 1992, Phys. Rev. D 45 n. 11.

Rosa, L., 1993, "Neutrino Masses and Proton Lifetime in SO(10) GUT Models" Ph. D. Thesis (unpublished).

Schramm, D., 1992, Nucl. Phys. B (Proc. Suppl.) 28 A, 243.

Shafi, Q., and C. Wetterich, 1979, Phys. Lett. B 85, 52.

Tuzi, T., 1989, "Two-loop Contributions to Spontaneous Breaking Scales in SO(10)" Ph. D.

Thesis (unpublished).

Weinberg, S., 1980, Phys. Lett. B 91, 51.

Yanagida, T., 1979, Proceedings of the Workshop on the Unified Theory and the Baryon 
Number of the Universe edited by O. Sawada et al. (KEK).

le Yaouanc, A., L. Oliver, O. Pène, and J.C. Raynal, 1977, Phys. Lett. B 72, 53. 
This figure "fig1-1.png" is available in "png" format from: http://arxiv.org/ps/hep-ph/9405332v1 
This figure "fig1-2.png" is available in "png" format from: http://arxiv.org/ps/hep-ph/9405332v1 